\title{
Amplitude equations for coupled electrostatic waves in the limit of weak instability
}

\author{
John David Crawford \\ Department of Physics and Astronomy \\ University of Pittsburgh \\ Pittsburgh, PA 15260 \\ Edgar Knobloch \\ Department of Physics \\ University of California \\ Berkeley, CA 94720
}

\begin{abstract}
We consider the simplest instabilities involving multiple unstable electrostatic plasma waves corresponding to four-dimensional systems of mode amplitude equations. In each case the coupled amplitude equations are derived up to third order terms. The nonlinear coefficients are singular in the limit in which the linear growth rates vanish together. These singularities are analyzed using techniques developed in previous studies of a single unstable wave. In addition to the singularities familiar from the one mode problem, there are new singularities in coefficients coupling the modes. The new singularities are most severe when the two waves have the same linear phase velocity and satisfy the spatial resonance condition $k_{2}=2 k_{1}$. As a result the short wave mode saturates at a dramatically smaller amplitude than that predicted for the weak growth rate regime on the basis of single mode theory. In contrast the long wave mode retains the single mode scaling. If these resonance conditions are not satisfied both modes retain their single mode scaling and saturate at comparable amplitudes.
\end{abstract}

August 21, 1997 


\section{Contents}

$\begin{array}{lll}1 & \text { Introduction } & 1\end{array}$

1.1 Notation . . . . . . . . . . . . . . . . . . . . . 4

1.2 Summary of linear theory . . . . . . . . . . . . . . . . . . 5

2 Amplitude equations: general features $\quad 6$

2.1 Instability without reflection symmetry: $F_{0}(v) \neq F_{0}(-v) \ldots$. . . . . . . . . 9

2.2 Instability with reflection symmetry: $F_{0}(v)=F_{0}(-v) \ldots \ldots$. . . . . . . . . 10

2.2.1 Real eigenvalues . . . . . . . . . . . . . . . . . . . . . . . . . . . . . . . . . .

2.2.2 Complex eigenvalues . . . . . . . . . . . . . . . . . . . . . . 11

2.3 Amplitude expansions . . . . . . . . . . . . . . . . . . . . . . . . . 12

2.4 Previous results for the single mode instabilities . . . . . . . . . . . . . . . . 15

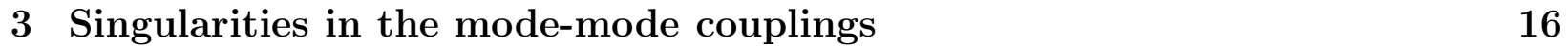

3.1 The universal couplings $p_{1}(0)$ and $r_{2}(0)$. . . . . . . . . . . . . . . . . 17

3.1.1 $F_{0}(v) \neq F_{0}(-v)$, complex eigenvalues . . . . . . . . . . . . . . . 18

3.1.2 $F_{0}(v)=F_{0}(-v)$, real eigenvalues . . . . . . . . . . . . . . . . 21

3.1.3 $F_{0}(v)=F_{0}(-v)$, complex eigenvalues . . . . . . . . . . . . . . . . . 22

3.2 The couplings $Q_{1}(0), P_{2}(0), Q_{3}(0)$ and $Q_{1}(0)+P_{3}(0)$. . . . . . . . . . . . . 23

3.3 The spatial resonances: $q(0)$ and $s(0)$. . . . . . . . . . . . . . . . 24

3.4 Special limits: coupling singularities with fixed ions . . . . . . . . . . . . . . 25

\begin{tabular}{lll}
\hline & Nonlinear scalings & 25
\end{tabular}

\begin{tabular}{lll}
\hline 5 Discussion & 30
\end{tabular}

\begin{tabular}{lll}
\hline$\quad$ Acknowledgements & 32
\end{tabular}

\section{Introduction}

Recently we presented a detailed analysis of the amplitude equation for a single unstable electrostatic mode in an unmagnetized Vlasov plasma (henceforth (I)). [1] The analysis reveals a fundamental difficulty with the derivation of amplitude equations for this class of problems: the coefficients in the amplitude equations become singular in the limit in which the growth rate $\gamma$ of the unstable wave is allowed to vanish. Although these singularities can be removed by an appropriate $\gamma$-dependent rescaling (see below) the analysis shows that amplitude equations of this type cannot be truncated at any finite order. [2, 3] Nonetheless the scaling 
identified by the theory predicts the amplitude at which weakly growing waves will saturate, and hence is of fundamental importance both in plasma physics and in the closely related problem of shear flow instability of ideal fluids.

In view of the importance of the predicted scaling for applications we investigate here the effects of including additional unstable modes. We consider only the simplest possibilities, those requiring a four-dimensional system of amplitude equations. There are three such instabilities distinguished by the symmetry of the equilibrium and whether the unstable modes have real or complex eigenvalues. We find that unless the two modes satisfy a strong resonance condition the presence of the second mode does not alter the saturation amplitude of the original mode. However, in the important resonant case in which the phase velocities of the two modes are the same and their wavenumbers $k_{1}, k_{2}$ satisfy $k_{2}=2 k_{1}$ the long wave mode suppresses dramatically the saturation amplitude of the short wave mode.

The amplitude equation defines, in the limit $\gamma \rightarrow 0^{+}$, a kind of singular perturbation problem whose detailed features reveal asymptotic scaling behavior of the nonlinear wave. This is a key idea behind our approach and the reader is referred to (I) for a more detailed discussion. For the single mode instabilities there is only one amplitude $A(t)$ and one seeks a scaling $A(t)=\gamma^{\beta} a(\gamma t)$ such that the evolution equation for $a(\tau), \tau \equiv \gamma t$, has a nonsingular limit as $\gamma \rightarrow 0^{+}$. In dissipative problems, the critical eigenvalues are isolated on the imaginary axis, and $\beta=1 / 2$ is the generically expected exponent. As a result, in the generic case, the amplitude equation can be truncated at third order. This is not so for an unstable electrostatic wave. Here the situation is quite different because the Vlasov equation is Hamiltonian and the eigenvalues of the mode merge with a continuous spectrum on the imaginary axis at criticality, i.e. as $\gamma \rightarrow 0^{+}$. As a consequence the nonlinear coefficients in the amplitude equation are singular as $\gamma \rightarrow 0^{+}$signalling strong nonlinear effects that saturate the unstable linear growth at exceptionally small wave amplitudes. A quantitative 
signature of this reduction of the nonlinear wave amplitude is a larger exponent: $\beta=5 / 2$ for plasmas with multiple mobile species and $\beta=2$ in the limiting case of infinitely massive (fixed) ions and mobile electrons. In fact, the analysis presented in (I) showed that setting $\beta=5 / 2$ yielded a theory that was finite to all orders in the amplitude expansion as $\gamma \rightarrow 0^{+}$.

In this paper we investigate the coupled amplitude equations for two unstable modes with amplitudes $A(t)$ and $B(t)$. The coupled equations contain the single mode instabilities due to excitation of only $A$ or only $B$, and the previously studied singular coefficients govern these special cases. There are now separate scalings possible for each amplitude,

$$
|A(t)|=\gamma_{1}^{\beta_{1}} a\left(\gamma_{1} t\right) \quad|B(t)|=\gamma_{2}^{\beta_{2}} b\left(\gamma_{2} t\right)
$$

and we know $\beta_{j} \geq 5 / 2, j=1,2$ is required to control the singularities in the single mode coefficients. We seek to determine the possible singularities in the coupling coefficients between $A$ and $B$ that are new; specifically we wish to know if these singularities can dominate the single mode singularities and require new nonlinear scalings for the instability with two simultaneously growing waves.

In the remainder of this introduction, we summarize our notation and state some relevant results about the linearized theory. Section 2 enumerates the possible instabilities described by four-dimensional systems of amplitude equations. In each case the general form of the amplitude equations can be anticipated on the basis of symmetry. Section 2.3 describes the procedure for calculating the coefficients in these amplitude equations and summarizes the results for the leading terms through third order. The singularities that arise in these terms in the limit of weak instability are analyzed in Section 3, and their consequences are discussed in Section 4. The paper ends with a brief conclusion. 


\subsection{Notation}

We briefly summarize the notation of (I) which will be used. Our model is the onedimensional, multi-species Vlasov plasma defined by

$$
\frac{\partial F^{(s)}}{\partial t}+v \frac{\partial F^{(s)}}{\partial x}+\kappa^{(s)} E \frac{\partial F^{(s)}}{\partial v}=0, \quad \frac{\partial E}{\partial x}=\sum_{s} \int_{-\infty}^{\infty} d v F^{(s)}(x, v, t) .
$$

Here $x, t$ and $v$ are measured in units of $u / \omega_{e}, \omega_{e}^{-1}$ and $u$, respectively, where $u$ is a chosen velocity scale and $\omega_{e}^{2}=4 \pi e^{2} n_{e} / m_{e}$. The plasma length is $L$ with periodic boundary conditions and

$$
\int_{-L / 2}^{L / 2} d x \int_{-\infty}^{\infty} d v F^{(s)}(x, v, t)=\left(\frac{z_{s} n_{s}}{n_{e}}\right) L
$$

where $q_{s}=e z_{s}$ is the charge of species $s$ and $\kappa^{(s)} \equiv q_{s} m_{e} / e m_{s}$. Note that $\kappa^{(e)}=-1$ for

electrons and that the normalization (3) for negative species makes the distribution function negative.

Let $F_{0}(v)$ and $f(x, v, t)$ denote the multi-component fields for the equilibrium and perturbation, respectively, and $\kappa$ the matrix of mass ratios,

$$
f \equiv\left(\begin{array}{c}
f^{\left(s_{1}\right)} \\
f^{\left(s_{2}\right)} \\
\vdots
\end{array}\right) \quad F_{0} \equiv\left(\begin{array}{c}
F_{0}^{\left(s_{1}\right)} \\
F_{0}^{\left(s_{2}\right)} \\
\vdots
\end{array}\right) \quad \kappa \equiv\left(\begin{array}{cccc}
\kappa^{\left(s_{1}\right)} & 0 & 0 & \cdots \\
0 & \kappa^{\left(s_{2}\right)} & 0 & \cdots \\
\vdots & \vdots & \vdots &
\end{array}\right)
$$

then the system (2) can be concisely expressed as

$$
\frac{\partial f}{\partial t}=\mathcal{L} f+\mathcal{N}(f)
$$

where

$$
\begin{aligned}
\mathcal{L} f & =\sum_{l=-\infty}^{\infty} e^{i l x}\left(L_{l} f_{l}\right)(v) \\
\left(L_{l} f_{l}\right)(v) & =\left\{\begin{array}{cc}
0 & l=0 \\
-i l\left[v f_{l}(v)+\kappa \cdot \eta_{l}(v) \sum_{s^{\prime}} \int_{-\infty}^{\infty} d v^{\prime} f_{l}^{\left(s^{\prime}\right)}\left(v^{\prime}\right)\right] & l \neq 0
\end{array}\right.
\end{aligned}
$$


with $\eta_{l}(v) \equiv-\partial_{v} F_{0} / l^{2}$, and

$$
\mathcal{N}(f)=\sum_{m=-\infty}^{\infty} e^{i m x} \sum_{l=-\infty}^{\infty}{ }^{\prime} \frac{i}{l}\left(\kappa \cdot \frac{\partial f_{m-l}}{\partial v}\right) \sum_{s^{\prime}} \int_{-\infty}^{\infty} d v^{\prime} f_{l}^{\left(s^{\prime}\right)}\left(v^{\prime}\right) .
$$

In the spatial Fourier expansion (6),$l$ denotes an integer multiple of the basic wavenumber $2 \pi / L$, and a primed summation as in (8) omits the $l=0$ term. The notation $\kappa \cdot \eta_{l}(v)$ or $\kappa \cdot \partial_{v} f_{m-l}$ denotes matrix multiplication.

An inner product is needed in Section 2 to derive the amplitude equation. For two multicomponent fields of $(x, v)$, e.g. $B=\left(B^{\left(s_{1}\right)}, B^{\left(s_{2}\right)}, B^{\left(s_{3}\right)}, ..\right)$ and $D=\left(D^{\left(s_{1}\right)}, D^{\left(s_{2}\right)}, D^{\left(s_{3}\right)}, ..\right)$, we define their inner product by

$$
(B, D) \equiv \sum_{s} \int_{-L / 2}^{L / 2} d x \int_{-\infty}^{\infty} d v B^{(s)}(x, v)^{*} D^{(s)}(x, v)=\int_{-L / 2}^{L / 2} d x<B, D>
$$

where

$$
<B, D>\equiv \sum_{s} \int_{-\infty}^{\infty} d v B^{(s)}(x, v)^{*} D^{(s)}(x, v)
$$

\subsection{Summary of linear theory}

The spectral theory for $\mathcal{L}$ is well established, and the needed results are simply recalled to

establish our notation. [4, 5, 6] The eigenvalues $\lambda \equiv-i l z$ of $\mathcal{L}$ are determined by the roots $\Lambda_{l}(z)=0$ of the "spectral function",

$$
\Lambda_{l}(z) \equiv 1+\int_{-\infty}^{\infty} d v \frac{\sum_{s} \kappa^{(s)} \eta_{l}^{(s)}(v)}{v-z}
$$

The linear dielectric function $\epsilon_{l}(z)$ is obtained on replacing the contour in (11) by the Landau contour for $\operatorname{Im}(z)<0$; for $\operatorname{Im}(z)>0, \Lambda_{l}(z)$ and $\epsilon_{l}(z)$ are the same function.

Associated with an eigenvalue $\lambda \equiv-i l z$ is the multi-component eigenfunction $\Psi(x, v)=$ $e^{i l x} \psi(v)$, where

$$
\psi(v)=-\frac{\kappa \cdot \eta_{l}}{v-z}
$$


There is also an associated adjoint eigenfunction $\tilde{\Psi}(x, v)=e^{i l x} \tilde{\psi}(v) / L$ satisfying $(\tilde{\Psi}, \Psi)=1$ with

$$
\tilde{\psi}(v)=-\frac{1}{\Lambda_{l}^{\prime}(z)^{*}\left(v-z^{*}\right)} .
$$

Note that all components of $\tilde{\psi}(v)$ are the same. The normalization in (13) assumes that the root of $\Lambda_{l}(z)$ is simple and is chosen so that $\langle\tilde{\psi}, \psi\rangle=1$. The adjoint determines the projection of $f(x, v, t)$ onto the eigenvector, and this projection defines the time-dependent amplitude of $\Psi$, i.e. $A(t) \equiv(\tilde{\Psi}, f)$.

In section 2.3 some of our results are conveniently stated in terms of the resolvent operator, $R_{l}(w) \equiv\left(w-L_{l}\right)^{-1}$, whose general form follows from (7) by solving $\left(w-L_{l}\right) f=g$ for $f$. [1], 6] Here both $g(v)=\left(g^{\left(s_{1}\right)}(v), g^{\left(s_{2}\right)}(v), \ldots\right)$ and $f$ are multi-component fields, and $R_{l}(w)$ acts by

$$
R_{l}(w) g=\left(\begin{array}{c}
\left(R_{l}(w) g\right)^{\left(s_{1}\right)}(v) \\
\left(R_{l}(w) g\right)^{\left(s_{2}\right)}(v) \\
\vdots
\end{array}\right)
$$

where

$$
\left(R_{l}(w) g\right)^{(s)}(v)=\frac{1}{i l(v-i w / l)}\left[g^{(s)}(v)-\frac{\kappa^{(s)} \eta_{l}^{(s)}}{\Lambda_{l}(i w / l)} \sum_{s^{\prime}} \int_{-\infty}^{\infty} d v^{\prime} \frac{g^{\left(s^{\prime}\right)}\left(v^{\prime}\right)}{v^{\prime}-i w / l}\right] .
$$

\section{Amplitude equations: general features}

Each of our instabilities can be formulated within a general framework as follows. The wavenumbers of the unstable modes $\Psi_{2}(x, v)$ and $\Psi_{1}(x, v)$ are given by $\left|k_{2}\right| \geq\left|k_{1}\right|>0$, and the corresponding eigenvalues are $\lambda_{j}=-i k_{j} z_{j}$ for $j=1,2$, where $\Lambda_{k_{j}}\left(z_{j}\right)=0$. With periodic boundary conditions, each wavenumber is a multiple of the minimum $k$, i.e. $k_{j}=2 \pi n_{j} / L$ with integer $n_{j}$. We assume all roots are simple, i.e. $\Lambda_{k_{j}}^{\prime}\left(z_{j}\right) \neq 0$; in addition, in the limit $\operatorname{Im}(z) \rightarrow 0$ of weak growth rates, the equation for the root is given by

$$
\sum_{s} \kappa^{(s)} \eta_{k_{j}}^{(s)}\left(v_{j}\right)=0, \quad 1+\mathrm{P} \int_{-\infty}^{\infty} d v \frac{\sum_{s} \kappa^{(s)} \eta_{k_{j}}^{(s)}(v)}{v-v_{j}}=0,
$$


where $v_{j}=\operatorname{Re}\left(z_{j}\right)$ is the phase velocity at criticality. These relations will be important for our analysis of the singularities in the nonlinear coefficients.

The four-dimensional eigenspace $E^{u}$ is spanned by $\left\{\Psi_{1}, \Psi_{1}^{*}, \Psi_{2}, \Psi_{2}^{*}\right\}$, and the components of the distribution function along the unstable eigenvectors are identified by writing

$$
f(x, v, t)=\left[A(t) \Psi_{1}(x, v)+B(t) \Psi_{2}(x, v)+c c\right]+S(x, v, t)
$$

where $A(t)=\left(\tilde{\Psi}_{1}, f\right)$ and $B(t)=\left(\tilde{\Psi}_{2}, f\right)$ are the mode amplitudes and $\left(\tilde{\Psi}_{j}, S\right)=0$. In (17), $\tilde{\Psi}_{j}=\exp \left(i k_{j} x\right) \tilde{\psi}_{j} / L$ is the appropriate adjoint function for $z_{j}$ from (13).

In the $(A, B, S)$ variables, the Vlasov equation (5) becomes:

$$
\begin{aligned}
\dot{A} & =\lambda_{1} A+\left(\tilde{\Psi}_{1}, \mathcal{N}(f)\right) \\
\dot{B} & =\lambda_{2} B+\left(\tilde{\Psi}_{2}, \mathcal{N}(f)\right) \\
\frac{\partial S}{\partial t} & =\mathcal{L} S+\mathcal{N}(f)-\left[\left(\tilde{\Psi}_{1}, \mathcal{N}(f)\right) \Psi_{1}+\left(\tilde{\Psi}_{2}, \mathcal{N}(f)\right) \Psi_{2}+c c\right]
\end{aligned}
$$

where

$$
\left(\tilde{\Psi}_{j}, \mathcal{N}(f)\right)=-i \sum_{l=-\infty}^{\infty} \frac{1}{l}<\partial_{v} \tilde{\psi}_{j}, \kappa \cdot f_{k_{j}-l}>\sum_{s^{\prime}} \int_{-\infty}^{\infty} d v^{\prime} f_{l}^{\left(s^{\prime}\right)}\left(v^{\prime}\right) .
$$

In writing (18) we have used the adjoint relationship $\left(\tilde{\Psi}_{j}, \mathcal{L} S\right)=\left(\mathcal{L}^{\dagger} \tilde{\Psi}_{j}, S\right)=\lambda_{j}\left(\tilde{\Psi}_{j}, S\right)=0$ and in (21) an integration by parts shifts the velocity derivative onto $\tilde{\psi}$.

These coupled equations are equivalent to (5); however, by restricting them to the unstable manifold we obtain autonomous equations for $A(t)$ and $B(t)$. The details of this restriction are discussed in an earlier paper[3] and also in (I). The unstable manifold is tangent to $E^{u}$ at the equilibrium, and near $F_{0}$ it can be described by a function $H\left(x, v, A, A^{*}, B, B^{*}\right)$. Thus

$$
f^{u}(x, v, t)=\left[A(t) \Psi_{1}(x, v)+B(t) \Psi_{2}(x, v)+c c\right]+H\left(x, v, A(t), A^{*}(t), B(t), B^{*}(t)\right)
$$


represents a distribution function on $W^{u}$. In this expression, the evolution of $S$ is determined from $H$, i.e.

$$
S(x, v, t)=H\left(x, v, A(t), A^{*}(t), B(t), B^{*}(t)\right)=\left(\begin{array}{c}
H^{\left(s_{1}\right)}\left(x, v, A, A^{*}, B, B^{*}\right) \\
H^{\left(s_{2}\right)}\left(x, v, A, A^{*}, B, B^{*}\right) \\
\vdots
\end{array}\right) .
$$

When this representation is substituted into (18) - (20) we obtain

$$
\begin{aligned}
\dot{A} & =\lambda_{1} A+\left(\tilde{\Psi}_{1}, \mathcal{N}\left(f^{u}\right)\right) \\
\dot{B} & =\lambda_{2} B+\left(\tilde{\Psi}_{2}, \mathcal{N}\left(f^{u}\right)\right) \\
\left.\frac{\partial S}{\partial t}\right|_{f^{u}} & =\mathcal{L} H+\mathcal{N}\left(f^{u}\right)-\left[\left(\tilde{\Psi}_{1}, \mathcal{N}\left(f^{u}\right)\right) \Psi_{1}+\left(\tilde{\Psi}_{2}, \mathcal{N}\left(f^{u}\right)\right) \Psi_{2}+c c\right] .
\end{aligned}
$$

Note that equations (24) - (25) define an autonomous flow describing the self-consistent nonlinear evolution of the unstable modes; this is the four-dimensional system we study.

Symmetries of the Vlasov-Poisson system (50) and the equilibrium $F_{0}(v)$ are important qualitative features of the problem. Spatial translation, $\mathcal{T}_{a}:(x, v) \rightarrow(x+a, v)$, and reflection, $\mathcal{R}:(x, v) \rightarrow(-x,-v)$, act as operators on $f(x, v, t)$ in the usual way: if $\alpha$ denotes an arbitrary transformation then $(\alpha f)(x, v) \equiv f\left(\alpha^{-1}(x, v)\right)$, where $(\alpha f)(x, v)$ denotes the transformed distribution function. The operators $\mathcal{L}$ and $\mathcal{N}$ commute with $\mathcal{T}_{a}$ due to the spatial homogeneity of $F_{0}$, and if $F_{0}(v)=F_{0}(-v)$, then $\mathcal{L}$ and $\mathcal{N}$ also commute with the reflection operator $\mathcal{R}$. With periodic boundary conditions, $x$ is a periodic coordinate so $\mathcal{T}_{a}$ and $\mathcal{R}$ generate $\mathrm{O}(2)$, the symmetry group of the circle. If only the translation symmetry is present, then the group is $\mathrm{SO}(2)$.

The action of translation $\mathcal{T}_{a}$ on $f(x, v, t)$ implies an action on the variables $(A, B, S)$. From (17) we note that $\mathcal{T}_{a} f(x, v, t)=f(x-a, v, t)$ is equivalent to

$$
\mathcal{T}_{a}(A, B, S(x, v))=\left(e^{-i k_{1} a} A, e^{-i k_{2} a} B, S(x-a, v)\right) .
$$

The representation of $\mathcal{R}$ in the variables $(A, B, S)$ depends on specific details of $\Psi_{1}$ and $\Psi_{2}$ 
in the individual cases discussed below. These symmetries determine the general form of the amplitude equations (24) - (25) for each of the instabilities we consider.

\subsection{Instability without reflection symmetry: $F_{0}(v) \neq F_{0}(-v)$}

When $F_{0}(v)$ lacks reflection symmetry, the generic four-dimensional problem arises for modes with unequal wavenumbers $k_{2}>k_{1}>0$ and complex eigenvalues. The roots, $\Lambda_{k_{j}}\left(z_{j}\right)=0$, determine the phase velocities $v_{j}=\omega_{j} / k_{j}$ and the growth rates $\gamma_{j}$ of the linear modes from the real and imaginary parts of the eigenvalue $\lambda_{j}=-i k_{j} z_{j} \equiv \gamma_{j}-i \omega_{j}$. The corresponding eigenvectors are

$$
\Psi_{j}(x, v)=e^{i k_{j} x} \psi_{j}(v)=e^{i k_{j} x}\left(-\frac{\kappa \cdot \eta_{k_{j}}}{v-z_{j}}\right), \quad j=1,2
$$

In this case, the identities $\Lambda_{k_{j}}\left(z_{j}\right)=\Lambda_{-k_{j}}\left(z_{j}\right)$ and $\Lambda_{k_{j}}\left(z_{j}\right)^{*}=\Lambda_{k_{j}}\left(z_{j}^{*}\right)$ imply three additional eigenvectors: $\Psi_{j}^{*}, \Phi_{j}$ and $\Phi_{j}^{*}$, where

$$
\Phi_{j}(x, v)=e^{i k_{j} x} \psi_{j}(v)^{*} .
$$

These eigenfunctions correspond to eigenvalues $\lambda_{j}^{*},-\lambda_{j}^{*}$ and $-\lambda_{j}$, respectively, and fill out the eigenvalue quartets characteristic of Hamiltonian systems. In the absence of reflection symmetry, the eigenvalues are typically simple and the four-dimensional unstable subspace is spanned by $\left\{\Psi_{1}, \Psi_{1}^{*}, \Psi_{2}, \Psi_{2}^{*}\right\}$. A beam-plasma system with a weak beam is the prototypical example of this instability.

Since $F_{0}(v)$ is spatially homogeneous, the amplitude equations (24) - (25) always have translation symmetry (27) and we can apply standard results on the form of such symmetric equations. [7, 8] Hence we know the right hand side of (24) - (25) takes the general form,

$$
\begin{aligned}
& \dot{A}=r(\sigma) A+s(\sigma)\left(A^{*}\right)^{n_{2}-1} B^{n_{1}} \\
& \dot{B}=p(\sigma) B+q(\sigma) A^{n_{2}}\left(B^{*}\right)^{n_{1}-1},
\end{aligned}
$$


where $\sigma=\left(\sigma_{1}, \sigma_{2}, \sigma_{3}, \sigma_{4}\right)$ denotes the four basic invariants

$$
\sigma_{1}=|A|^{2} \quad \sigma_{2}=|B|^{2} \quad \sigma_{3}=A^{n_{2}}\left(B^{*}\right)^{n_{1}} \quad \sigma_{4}=\left(A^{*}\right)^{n_{2}} B^{n_{1}} .
$$

The integers $n_{j}$ refer to the wavenumbers $k_{j}=2 \pi n_{j} / L$. [9] The complex-valued functions $r, s, p$, and $q$ are determined from (24) - (25), but they can depend on the amplitudes only through the four invariants $\sigma_{1}, \sigma_{2}, \sigma_{3}$ and $\sigma_{4}$.

\subsection{Instability with reflection symmetry: $F_{0}(v)=F_{0}(-v)$}

When the equilibrium is reflection-symmetric the transformation $\mathcal{R} f(x, v, t)=f(-x,-v, t)$ commutes with $\mathcal{L}$ and $\mathcal{N}$ in the Vlasov equation. In this setting, the roots of $\Lambda_{l}(z)$ can be either imaginary or complex, depending on the detailed form of $F_{0}(v)$, and correspondingly we may encounter instabilities due to either real or complex eigenvalues.

\subsubsection{Real eigenvalues}

The description for real eigenvalues is quite similar to the examples without symmetry and we use the same notation (28) for the unstable eigenvectors $\Psi_{1}(x, v)$ and $\Psi_{2}(x, v)$. In this case, however, both linear phase velocities are zero. In addition, each of the real eigenvalues $\lambda_{1}$ and $\lambda_{2}$ has multiplicity two since the states $\mathcal{R} \Psi_{1}=\Psi_{1}^{*}$ and $\mathcal{R} \Psi_{2}=\Psi_{2}^{*}$ are also eigenvectors. The translation symmetry (27) still holds, as well as the reflection transformation given by

$$
\mathcal{R}(A, B, S(x, v))=\left(A^{*}, B^{*}, S(-x,-v)\right) .
$$

The form of the amplitude equations (24) - (25) is the same except that the functions $r, s, p$ and $q$ in (30) - (31) are now real-valued and depend on only three invariants $\sigma_{1}, \sigma_{2}$, and $\sigma_{+} \equiv \sigma_{3}+\sigma_{4}$. This model applies for example to a reflection-symmetric two-stream distribution with instability at two wavenumbers. 


\subsubsection{Complex eigenvalues}

An instability in a reflection-symmetric system with complex eigenvalues likewise yields eigenvalues of multiplicity two. If only one wavelength is involved these result in a fourdimensional problem, and provide an additional example of what may be called a one-mode instability, cf. (I). Such an instability arises, for example, in a beam-plasma system with counter-propagating beams. [10]

In the notation of our general framework, we let $k_{1}=-k_{2}=k>0$ and $z_{1}=-z_{2}=z_{0}$; then $\Lambda_{k}\left(z_{0}\right)=0$ implies $\Lambda_{-k}\left(-z_{0}\right)=0$, and these roots correspond to reflection-related eigenvectors for the eigenvalue $\lambda=-i k z_{0}$, i.e. $\lambda=-i k_{1} z_{1}=-i k_{2} z_{2}$ :

$$
\begin{aligned}
& \Psi_{1}(x, v)=e^{i k x} \psi_{1}(v)=e^{i k x}\left(-\frac{\kappa \cdot \eta_{k}(v)}{v-z_{0}}\right) \\
& \Psi_{2}(x, v)=\left(\mathcal{R} \Psi_{1}\right)(x, v)=e^{-i k x} \psi_{1}(-v)=e^{-i k x}\left(-\frac{\kappa \cdot \eta_{k}(v)}{v+z_{0}}\right) .
\end{aligned}
$$

These solutions describe oppositely propagating linear waves with phase velocities $v_{1}=$ $-v_{2}=\omega / k$, where $z_{0}=v_{1}+i \gamma / k$. In contrast to the previous examples, where both $z_{1}$ and $z_{2}$ sit in the upper-half plane (corresponding to positive wavenumbers), in this case $z_{2}$ falls in the lower-half plane.

Now the mode amplitudes in (17) transform according to (27) and applying $\mathcal{R}$ to (17) yields

$$
\mathcal{R}(A, B, S(x, v))=(B, A, S(-x,-v)) .
$$

The $\mathrm{O}(2)$ symmetry generated by $\mathcal{R}$ and $\mathcal{T}_{a}$ implies amplitude equations of the form

$$
\left(\begin{array}{c}
\dot{A} \\
\dot{B}
\end{array}\right)=P\left(\mu_{1}, \mu_{2}, \mu_{2}^{*}\right)\left(\begin{array}{c}
A \\
B
\end{array}\right)+Q\left(\mu_{1}, \mu_{2}, \mu_{2}^{*}\right)\left(\begin{array}{c}
B^{*} \\
A^{*}
\end{array}\right),
$$

where $P$ and $Q$ are functions of the invariants $\mu_{1} \equiv|A|^{2}+|B|^{2}$ and $\mu_{2}=A B$ with $P(0,0,0)=$ $\lambda$ and $Q(0,0,0)=0$. In this case a further simplification is possible; terms that do not 
commute with the "phase-shift" symmetry, $(A, B) \rightarrow\left(e^{i \phi} A, e^{i \phi} B\right)$, can be removed by nearidentity coordinate changes, $(A, B) \equiv\left(A^{\prime}+\Phi_{1}\left(A^{\prime}, B^{\prime}\right), B^{\prime}+\Phi_{2}\left(A^{\prime}, B^{\prime}\right)\right)$, to obtain the normal form:

$$
\left(\begin{array}{c}
\dot{A} \\
\dot{B}
\end{array}\right)=R\left(\mu_{1}, \mu_{3}\right)\left(\begin{array}{c}
A \\
B
\end{array}\right)+S\left(\mu_{1}, \mu_{3}\right)\left[|A|^{2}-|B|^{2}\right]\left(\begin{array}{c}
A \\
-B
\end{array}\right)
$$

where $\mu_{3} \equiv\left(|A|^{2}-|B|^{2}\right)^{2}$ and we have dropped the primes on $\left(A^{\prime}, B^{\prime}\right)$. [0, 8]

\subsection{Amplitude expansions}

We wish to study the nonlinear terms (21) in the amplitude equations (24) - (25). The Fourier components $f_{l}$ follow from (22)

$$
f_{l}^{u}(v)=A \psi_{1}(v) \delta_{l, k_{1}}+A^{*} \psi_{1}(v)^{*} \delta_{l,-k_{1}}+B \psi_{2}(v) \delta_{l, k_{2}}+B^{*} \psi_{2}(v)^{*} \delta_{l,-k_{2}}+H_{l}(v),
$$

and the amplitude expansion of $H_{l}(v)$ begins with second-order terms,

$$
\begin{aligned}
H_{l}(v)= & {\left[h_{1}(v)|A|^{2}+h_{2}(v)|B|^{2}\right] \delta_{l, 0}+h_{3}(v) B A^{*} \delta_{l, k_{2}-k_{1}}+h_{3}(v)^{*} B^{*} A \delta_{l, k_{1}-k_{2}} } \\
& +h_{4}(v) A^{2} \delta_{l, 2 k_{1}}+h_{4}(v)^{*}\left(A^{*}\right)^{2} \delta_{l,-2 k_{1}}+h_{5}(v) A B \delta_{l, k_{2}+k_{1}}+h_{5}(v)^{*} A^{*} B^{*} \delta_{l,-k_{2}-k_{1}} \\
& +h_{6}(v) B^{2} \delta_{l, 2 k_{2}}+h_{6}(v)^{*}\left(B^{*}\right)^{2} \delta_{l,-2 k_{2}}+\mathcal{O}(3) .
\end{aligned}
$$

Thus the nonlinear terms (21) can be written out in terms of the coefficients $h_{i}(v)$ in (40), neglecting terms that are higher than third order in the amplitudes:

$$
\begin{aligned}
& \left(\tilde{\Psi}_{1}, \mathcal{N}\left(f^{u}\right)\right)=r_{1}(0) A|A|^{2}+r_{2}(0) A|B|^{2}+\delta_{k_{2}, 2 k_{1}} s(0) A^{*} B+\delta_{k_{2}, 3 k_{1}} s(0)\left(A^{*}\right)^{2} B \\
& \quad+\delta_{k_{2},-k_{1}}\left[P_{2}(0) A^{2} B+\left(Q_{1}(0)+P_{3}(0)\right) B^{*}|A|^{2}+Q_{3}(0) A^{*}\left(B^{*}\right)^{2}+Q_{1}(0) B^{*}|B|^{2}\right]
\end{aligned}
$$

with Taylor coefficients

$$
r_{1}(0)=-\frac{i}{k_{1}}\left[<\partial_{v} \tilde{\psi}_{1}, \kappa \cdot\left(h_{1}-h_{4}\right)>+\frac{\Gamma_{4}}{2}<\partial_{v} \tilde{\psi}_{1}, \kappa \cdot \psi_{1}^{*}>\right]
$$




$$
\begin{aligned}
& r_{2}(0)=-i\left[\frac{<\partial_{v} \tilde{\psi}_{1}, \kappa \cdot h_{2}>}{k_{1}}+\frac{<\partial_{v} \tilde{\psi}_{1}, \kappa \cdot\left(h_{3}^{*}-h_{5}\right)>}{k_{2}}-\frac{\Gamma_{3}^{*}}{k_{2}-k_{1}}<\partial_{v} \tilde{\psi}_{1}, \kappa \cdot \psi_{2}>\right. \\
& \left.+\frac{\Gamma_{5}}{k_{1}+k_{2}}<\partial_{v} \tilde{\psi}_{1}, \kappa \cdot \psi_{2}^{*}>\right] \\
& s(0)=\left\{\begin{array}{c}
-i\left[<\partial_{v} \tilde{\psi}_{1}, \kappa \cdot \psi_{1}^{*}>/ k_{2}-<\partial_{v} \tilde{\psi}_{1}, \kappa \cdot \psi_{2}>/ k_{1}\right] \\
-i\left[<\partial_{v} \tilde{\psi}_{1}, \kappa \cdot h_{4}^{*}>/ k_{2}-<\partial_{v} \tilde{\psi}_{1}, \kappa \cdot h_{3}>/ k_{1}\right. \\
+\Gamma_{3}<\partial_{v} \tilde{\psi}_{1}, \kappa \cdot \psi_{1}^{*}>/\left(k_{2}-k_{1}\right) \\
\left.-\Gamma_{4}^{*}<\partial_{v} \tilde{\psi}_{1}, \kappa \cdot \psi_{2}>/ 2 k_{1}\right]
\end{array} \quad \text { if } \quad k_{2}=3 k_{1}\right. \\
& P_{2}(0)=-i\left[\frac{<\partial_{v} \tilde{\psi}_{1}, \kappa \cdot h_{5}>}{k_{1}}+\frac{<\partial_{v} \tilde{\psi}_{1}, \kappa \cdot h_{4}>}{k_{2}}+\frac{\Gamma_{4}}{2 k_{1}}<\partial_{v} \tilde{\psi}_{1}, \kappa \cdot \psi_{2}>\right] \\
& Q_{1}(0)+P_{3}(0)=-i\left[\frac{<\partial_{v} \tilde{\psi}_{1}, \kappa \cdot\left(h_{5}^{*}-h_{3}^{*}\right)>}{k_{1}}-\frac{<\partial_{v} \tilde{\psi}_{1}, \kappa \cdot h_{1}>}{k_{2}}\right. \\
& \left.+\frac{\Gamma_{3}^{*}<\partial_{v} \tilde{\psi}_{1}, \kappa \cdot \psi_{1}^{*}>}{k_{1}-k_{2}}\right] \\
& Q_{3}(0)=i\left[\frac{<\partial_{v} \tilde{\psi}_{1}, \kappa \cdot\left(h_{6}^{*}-h_{5}^{*}\right)>}{k_{1}}+\frac{\Gamma_{6}^{*}}{2 k_{2}}<\partial_{v} \tilde{\psi}_{1}, \kappa \cdot \psi_{1}^{*}>\right] \\
& Q_{1}(0)=-i\left[\frac{<\partial_{v} \tilde{\psi}_{1}, \kappa \cdot\left(h_{6}^{*}-h_{2}\right)>}{k_{2}}-\frac{\Gamma_{6}^{*}}{2 k_{2}}<\partial_{v} \tilde{\psi}_{1}, \kappa \cdot \psi_{2}>\right]
\end{aligned}
$$

where $\Gamma_{j} \equiv \sum_{s} \int d v h_{j}^{(s)}(v)$, and

$$
\begin{aligned}
& \left(\tilde{\Psi}_{2}, \mathcal{N}\left(f^{u}\right)\right)=p_{1}(0) B|A|^{2}+p_{2}(0) B|B|^{2}+\delta_{k_{2}, 2 k_{1}} q(0) A^{2}+\delta_{k_{2}, 3 k_{1}} q(0) A^{3} \\
& \quad+\delta_{k_{2},-k_{1}}\left[P_{2}(0) A B^{2}+\left(Q_{1}(0)+P_{3}(0)\right) A^{*}|B|^{2}+Q_{3}(0)\left(A^{*}\right)^{2} B^{*}+Q_{1}(0) A^{*}|A|^{2}\right]
\end{aligned}
$$

with coefficients

$$
\begin{gathered}
p_{1}(0)=-i\left[\frac{<\partial_{v} \tilde{\psi}_{2}, \kappa \cdot\left(h_{3}-h_{5}\right)>}{k_{1}}+\frac{<\partial_{v} \tilde{\psi}_{2}, \kappa \cdot h_{1}>}{k_{2}}+\frac{\Gamma_{3}}{k_{2}-k_{1}}<\partial_{v} \tilde{\psi}_{2}, \kappa \cdot \psi_{1}>\right. \\
\left.+\frac{\Gamma_{5}}{k_{2}+k_{1}}<\partial_{v} \tilde{\psi}_{2}, \kappa \cdot \psi_{1}^{*}>\right] \\
p_{2}(0)=-\frac{i}{k_{2}}\left[<\partial_{v} \tilde{\psi}_{2}, \kappa \cdot\left(h_{2}-h_{6}\right)>+\frac{\Gamma_{6}}{2}<\partial_{v} \tilde{\psi}_{2}, \kappa \cdot \psi_{2}^{*}>\right],
\end{gathered}
$$




$$
q(0)=\left\{\begin{array}{lll}
-i<\partial_{v} \tilde{\psi}_{2}, \kappa \cdot \psi_{1}>/ k_{1} & \text { if } & k_{2}=2 k_{1} \\
-i\left[<\partial_{v} \tilde{\psi}_{2}, \kappa \cdot h_{4}>/ k_{1}+\Gamma_{4}<\partial_{v} \tilde{\psi}_{2}, \kappa \cdot \psi_{1}>/ 2 k_{1}\right] & \text { if } & k_{2}=3 k_{1} .
\end{array}\right.
$$

In (41) and (49) we abbreviate the notation of Section 2 letting $r_{j}(0)$ denote $\partial_{\sigma_{j}} r(0), P_{j}(0)$ denote $\partial_{\mu_{j}} P(0)$ and so forth. For an instability with complex eigenvalues and a reflection symmetry, the wavenumbers satisfy $k_{2}=-k_{1}$, and the $\Gamma_{5}$ terms in $r_{2}(0)$ and $p_{1}(0)$ are omitted. In addition, the reflection symmetry (36) implies various identities: $r_{1}(0)=p_{2}(0)$, $r_{2}(0)=p_{1}(0), h_{1}(v)=h_{2}(-v), h_{3}(v)=h_{3}(-v)^{*}, h_{4}(v)=h_{6}(-v)$, and $h_{5}(v)=h_{5}(-v)$, and these relate the cubic terms in (41) and (49) as shown.

The coefficients $h_{i}(v)$ follow from the second-order terms in (26). On the left-hand side of (26), we have

$$
\left.\frac{\partial S}{\partial t}\right|_{f^{u}}=\left[\frac{\partial H}{\partial A} \dot{A}+\frac{\partial H}{\partial B} \dot{B}+c c\right]=\left[\frac{\partial H}{\partial A} \lambda_{1} A+\frac{\partial H}{\partial B} \lambda_{2} B+c c\right]+\mathcal{O}(3),
$$

with the partial derivatives evaluated using (40). On the right-hand side of (26), the leading terms are found in $\mathcal{L} H$ and

$$
\begin{aligned}
\mathcal{N}\left(f^{u}\right)= & \frac{i|A|^{2}}{k_{1}} \kappa \cdot \partial_{v}\left(\psi_{1}^{*}-\psi_{1}\right)+\frac{i|B|^{2}}{k_{2}} \kappa \cdot \partial_{v}\left(\psi_{2}^{*}-\psi_{2}\right) \\
+ & \left\{\frac{i A^{2}}{k_{1}} \kappa \cdot \partial_{v} \psi_{1} e^{i 2 k_{1} x}+\frac{i B^{2}}{k_{2}} \kappa \cdot \partial_{v} \psi_{2} e^{i 2 k_{2} x}+i A B\left(\frac{\kappa \cdot \partial_{v} \psi_{2}}{k_{1}}+\frac{\kappa \cdot \partial_{v} \psi_{1}}{k_{2}}\right) e^{i\left(k_{1}+k_{2}\right) x}\right. \\
& \left.+i A B^{*}\left(\frac{\kappa \cdot \partial_{v} \psi_{2}^{*}}{k_{1}}-\frac{\kappa \cdot \partial_{v} \psi_{1}}{k_{2}}\right) e^{i\left(k_{1}-k_{2}\right) x}+c c\right\}+\mathcal{O}(3) .
\end{aligned}
$$

The second-order solution of (26) determines the coefficients in (40):

$$
\begin{array}{ll}
h_{1}(v)=\frac{i \kappa \cdot \partial_{v}\left(\psi_{1}^{*}-\psi_{1}\right)}{2 \gamma_{1} k_{1}} & h_{2}(v)=\frac{i \kappa \cdot \partial_{v}\left(\psi_{2}^{*}-\psi_{2}\right)}{2 \gamma_{2} k_{2}} \\
h_{3}(v)=i R_{k_{2}-k_{1}}\left(\lambda_{1}^{*}+\lambda_{2}\right) I_{3}(v) & h_{4}(v)=i R_{2 k_{1}}\left(2 \lambda_{1}\right) I_{4}(v) \\
h_{5}(v)=i R_{k_{2}+k_{1}}\left(\lambda_{1}+\lambda_{2}\right) I_{5}(v) & h_{6}(v)=i R_{2 k_{2}}\left(2 \lambda_{2}\right) I_{6}(v)
\end{array}
$$

where $R_{l}(w)$ denotes the resolvent operator (15), and

$$
I_{3}(v) \equiv \frac{\kappa \cdot \partial_{v} \psi_{1}^{*}}{k_{2}}-\frac{\kappa \cdot \partial_{v} \psi_{2}}{k_{1}}+i s(0) \psi_{1} \delta_{k_{2}, 2 k_{1}} \quad I_{5}(v) \equiv \frac{\kappa \cdot \partial_{v} \psi_{1}}{k_{2}}+\frac{\kappa \cdot \partial_{v} \psi_{2}}{k_{1}}
$$




$$
I_{4}(v) \equiv \frac{\kappa \cdot \partial_{v} \psi_{1}}{k_{1}}+i q(0) \psi_{2} \delta_{k_{2}, 2 k_{1}} \quad I_{6}(v) \equiv \frac{\kappa \cdot \partial_{v} \psi_{2}}{k_{2}}
$$

These expressions are valid for each of the three instabilities we consider, with one exception. In the case of complex eigenvalues and reflection symmetry, when $k_{1}+k_{2}=0$, the result for $h_{5}(v)$ in (57) is replaced by

$$
h_{5}(v)=-\frac{I_{5}(v)}{2 k z_{0}} \quad\left(k_{1}+k_{2}=0\right)
$$

in the notation of Section 2.2.2.

Note that (55) implies $\Gamma_{1}=\Gamma_{2}=0$ in general, and (60) forces $\Gamma_{5}=0$ for this specific

type of instability. Following (I), we can re-express $\left(\Gamma_{j}, h_{j}\right), j=3,4,5,6$ in (55) - (57) more conveniently as

$$
\Gamma_{j}=\left(\frac{1 / l_{j}}{\Lambda_{l_{j}}\left(z_{j}\right)}\right) \int_{-\infty}^{\infty} \frac{d v \sum_{s} I_{j}^{(s)}(v)}{v-z_{j}} \quad h_{j}(v)=\frac{I_{j}(v)}{l_{j}\left(v-z_{j}\right)}-\Gamma_{j}\left(\frac{\kappa \cdot \eta_{l_{j}}(v)}{v-z_{j}}\right),
$$

where

$$
\begin{aligned}
& l_{3}=k_{2}-k_{1}, \quad z_{3}=\frac{k_{2} z_{2}-k_{1} z_{1}^{*}}{k_{2}-k_{1}} ; \quad l_{4}=2 k_{1}, \quad z_{4}=z_{1} ; \\
& l_{5}=k_{2}+k_{1}, \quad z_{5}=\frac{k_{2} z_{2}+k_{1} z_{1}}{k_{2}+k_{1}} ; \quad l_{6}=2 k_{2}, \quad z_{6}=z_{2} .
\end{aligned}
$$

\subsection{Previous results for the single mode instabilities}

For $A=0$ in (30) - (31), we recover the single mode instability $\dot{B}=p\left(|B|^{2}\right) B=\left[\lambda_{2}+\right.$ $\left.p_{2}(0)|B|^{2}+\cdots\right] B$ previously considered[1], 2]; the asymptotic form of the cubic coefficient is

$$
p_{2}(0)=\frac{1}{\gamma_{2}^{4}}\left[c\left(k_{2}, z_{2}\right)-\gamma_{2} d\left(k_{2}, z_{2}\right)+\mathcal{O}\left(\gamma_{2}^{2}\right)\right]
$$

with the nonsingular functions $c$ and $d$ defined by

$$
\begin{aligned}
c\left(k_{2}, z_{2}\right) & =-\frac{k_{2}}{4 \Lambda_{k_{2}}^{\prime}\left(z_{2}\right)} \sum_{s}^{\prime} \kappa^{(s)}\left(1-\kappa^{(s)^{2}}\right) \operatorname{Im}\left(\int_{-\infty}^{\infty} d v \frac{\eta_{k_{2}}^{(s)}}{v-z_{2}}\right) \\
d\left(k_{2}, z_{2}\right) & =\frac{1}{4}-\frac{1}{4 \Lambda_{k_{2}}^{\prime}\left(z_{2}\right)} \sum_{s}^{\prime} \kappa^{(s)}\left(1-\kappa^{(s)^{2}}\right) \int_{-\infty}^{\infty} d v \frac{\eta_{k_{2}}^{(s)}}{\left(v-z_{2}\right)^{2}}
\end{aligned}
$$


where the primed species sum omits the electrons. [1] For $n_{1}>1$ in (30) - (31), setting $B=0$ determines a single mode instability at the longer wavelength: $\dot{A}=r\left(|A|^{2}\right) A=$ $\left[\lambda_{1}+r_{1}(0)|A|^{2}+\cdots\right] A$. The asymptotic form of $r_{1}(0)$ follows from (64) - (66) with the replacements $\left(\gamma_{2}, k_{2}, z_{2}\right) \rightarrow\left(\gamma_{1}, k_{1}, z_{1}\right)$. We also reduce to this previously studied case on setting either $A=0$ or $B=0$ in equation (38); these two limits correspond to singlemode instabilities in the form of travelling waves propagating in the positive and negative $x$ directions, respectively.

\section{Singularities in the mode-mode couplings}

The central result of the single mode analysis proves that setting $\beta_{j}=5 / 2$ in (1) yields rescaled amplitude equations for $a(\tau)$ and $b(\tau)$ that are finite to all orders. [1] When there are two unstable modes additional coupling terms between the modes are present that have not been previously considered. In particular in the expansions (41) and (49) we find four such couplings, $\left(q(0), s(0), p_{1}(0), r_{2}(0)\right)$, for instabilities with $k_{2}>k_{1}>0$ (cf. Sections 2.1 2.2.1) and five couplings, $\left(p_{1}(0), Q_{1}(0), P_{2}(0), Q_{3}(0), Q_{1}(0)+P_{3}(0)\right)$, for an instability with $k_{2}+k_{1}=0$ (cf. Section 2.2.2). The singularities of such mode coupling terms will determine if the presence of a second unstable wave can alter the nonlinear scaling associated with single wave instabilities.

This question needs to be formulated carefully to avoid "trivial" limits since there are now two distinct linear growth rates $\gamma_{1}$ and $\gamma_{2}$. If one growth rate vanishes while the second remains bounded above zero, then only the singularities associated with the resonant denominators of the first mode will emerge. This effectively recovers the singularity structure of the one mode problem even though both mode amplitudes are non-zero. The more interesting limit arises when all resonant denominators come into play which requires both growth rates to vanish simultaneously. Thus we set $\gamma_{1}=\gamma_{2} \equiv \gamma$ in the following discussion; 
in practice this arrangement would be hard to realize experimentally but could be achieved in a numerical simulation by simultaneously adjusting the parameters of the equilibrium and the length of the system.

The origin of the singularities is the same as in the one mode problem: poles from resonant denominators can straddle the contour of integration and produce pinching singularities as $\gamma \rightarrow 0^{+}$. Following the methodology of (I), the worst possible singularity of a given integral can be estimated by simply counting the total number of poles (including multiplicity). This gives an upper bound on the possible divergence of a given integral which must be checked by a detailed evaluation once the most divergent integrals have been identified. Ultimately we are most interested in possible singularities that are stronger than those already identified in the single-mode subsystem (64). For example, third order terms with divergences weaker than the $\gamma^{-4}$ singularity of (64) are clearly sub-dominant and cannot alter the $\beta_{j}=5 / 2$ scaling forced by the single mode singularities.

In Sections 3.1 - 3.3, we assume the single mode problems exhibit the $\gamma^{-4}$ cubic singularity in (64), i.e.

$$
\sum_{s}^{\prime} \kappa^{(s)}\left(1-\kappa^{(s)^{2}}\right) \eta_{k_{j}}^{(s)}\left(v_{j}\right) \neq 0, \quad j=1,2
$$

for modes $k_{1}$ and $k_{2}$, respectively. This is simply the condition that in (65) $c\left(k_{j}, v_{j}+i 0\right) \neq 0$, $j=1,2$. Special limits, such as infinitely massive ions, for which the single mode system is less singular are discussed in Section 3.4. Tables 1 and 2 provide a summary of the asymptotic behavior of the second and third order coupling coefficients.

\subsection{The universal couplings $p_{1}(0)$ and $r_{2}(0)$}

The coefficients $r_{2}(0)$ and $p_{1}(0)$ are present at third order for each instability, and their asymptotic singularities are fundamental. We discuss the three types of instabilities separately. 


\subsection{1 $F_{0}(v) \neq F_{0}(-v)$, complex eigenvalues}

For instabilities without reflection symmetry (Section 2.1), we have $k_{2}+k_{1}>0$ for two positive and unequal wavenumbers. We first identify integrals in $r_{2}(0)$ and $p_{1}(0)$ with poles above and below the contour; terms without this feature are manifestly free of pinching singularities and will be finite as $\gamma \rightarrow 0^{+}$. In addition, singularities weaker than $\gamma^{-4}$ are sub-dominant.

From (62) - (63), $\operatorname{Im}\left(z_{j}\right) \geq 0$ for $j=1, \ldots, 6$ so $\psi_{1}(v), \psi_{2}(v)$ have poles only in the upper-

half plane as do the conjugated adjoints $\tilde{\psi}_{j}(v)^{*}$; thus $<\partial_{v} \tilde{\psi}_{1}, \kappa \cdot \psi_{2}>$ and $<\partial_{v} \tilde{\psi}_{2}, \kappa \cdot \psi_{1}>$ are nonsingular. Similarly, $I_{5}(v)$ and $h_{5}(v)$ have poles only in the upper-half plane, thus the integral in (61) for $\Gamma_{5}$ is nonsingular; in addition, $\Lambda_{l_{5}}\left(z_{5}\right)=\Lambda_{k_{2}+k_{1}}\left(z_{5}\right)$ is of order unity as $\gamma \rightarrow 0$ since there are no modes at wavenumber $k_{2}+k_{1}$ by assumption. Hence $\Gamma_{5}$ and $<\partial_{v} \tilde{\psi}_{1}, h_{5}>$ are both nonsingular. The integrals $<\partial_{v} \tilde{\psi}_{1}, \kappa \cdot \psi_{2}^{*}>$ and $<\partial_{v} \tilde{\psi}_{2}, \kappa \cdot \psi_{1}^{*}>$ are nonsingular unless $v_{1}=v_{2}$ in which case $\gamma^{-2}$ is their worst possible divergence, so the terms involving $\Gamma_{5}$ are sub-dominant.

Similarly in

$$
<\partial_{v} \tilde{\psi}_{1}, \kappa \cdot h_{2}>=\frac{i}{2 \gamma k_{2}}\left[<\partial_{v} \tilde{\psi}_{1}, \kappa^{2} \cdot \partial_{v} \psi_{2}^{*}>-<\partial_{v} \tilde{\psi}_{1}, \kappa^{2} \cdot \partial_{v} \psi_{2}>\right]
$$

the second integral is nonsingular and the first integral, $\left\langle\partial_{v} \tilde{\psi}_{1}, \kappa^{2} \cdot \partial_{v} \psi_{2}^{*}\right\rangle$, is nonsingular unless $v_{2}=v_{1}$ in which case there is a $\gamma^{-3}$ singularity. This conclusion applies equally to the corresponding terms in $\left\langle\partial_{v} \tilde{\psi}_{2}, \kappa \cdot h_{1}>\right.$; thus these terms contribute at most a $\gamma^{-4}$ singularity to $r_{2}(0)$ and $p_{1}(0)$, respectively.

Discarding these terms, we must still consider the asymptotic behavior due to $h_{3}$ and $\Gamma_{3}$ :

$$
\begin{aligned}
& r_{2}(0)=-i\left[\frac{<\partial_{v} \tilde{\psi}_{1}, \kappa \cdot h_{3}^{*}>}{k_{2}}-\frac{\Gamma_{3}^{*}}{k_{2}-k_{1}}<\partial_{v} \tilde{\psi}_{1}, \kappa \cdot \psi_{2}>\right]+\cdots \\
& p_{1}(0)=-i\left[\frac{<\partial_{v} \tilde{\psi}_{2}, \kappa \cdot h_{3}>}{k_{1}}+\frac{\Gamma_{3}}{k_{2}-k_{1}}<\partial_{v} \tilde{\psi}_{2}, \kappa \cdot \psi_{1}>\right]+\cdots,
\end{aligned}
$$


where

$$
\begin{aligned}
& \Gamma_{3}=\left(\frac{1 / l_{3}}{\Lambda_{l_{3}}\left(z_{3}\right)}\right) \int_{-\infty}^{\infty} \frac{d v \sum_{s}}{v-z_{3}}\left[\frac{\kappa^{(s)} \partial_{v} \psi_{1}^{(s)^{*}}}{k_{2}}-\frac{\kappa^{(s)} \partial_{v} \psi_{2}^{(s)}}{k_{1}}+i s(0) \psi_{1}^{(s)} \delta_{k_{2}, 2 k_{1}}\right] \\
&< \partial_{v} \tilde{\psi}_{1}, \kappa \cdot h_{3}^{*}>=<\partial_{v} \tilde{\psi}_{1}, \frac{\kappa \cdot I_{3}^{*}}{l_{3}\left(v-z_{3}^{*}\right)}>-\Gamma_{3}^{*}<\partial_{v} \tilde{\psi}_{1}, \frac{\kappa^{2} \cdot \eta_{l_{3}}}{\left(v-z_{3}^{*}\right)}> \\
&<\partial_{v} \tilde{\psi}_{2}, \kappa \cdot h_{3}>=<\partial_{v} \tilde{\psi}_{2}, \frac{\kappa \cdot I_{3}}{l_{3}\left(v-z_{3}\right)}>-\Gamma_{3}<\partial_{v} \tilde{\psi}_{2}, \frac{\kappa^{2} \cdot \eta_{l_{3}}}{\left(v-z_{3}\right)}>.
\end{aligned}
$$

For (69) - (70), we discuss the instabilities with $k_{2} \neq 2 k_{1}$ and $k_{2}=2 k_{1}$ separately; the latter case is more interesting since there can be new singularities when the modes have the same phase velocity, e.g. in a beam-plasma instability with a sufficiently cold beam. [17]

When $k_{2} \neq 2 k_{1}$, the third term in (71) is absent and the second term is manifestly nonsingular; from (62),

$$
z_{3}=\frac{k_{2} v_{2}-k_{1} v_{1}}{k_{2}-k_{1}}+i\left(\frac{2 \gamma}{k_{2}-k_{1}}\right)
$$

so the first term in (71) has a pinching singularity only if $v_{2}=v_{1}$ and this possibility yields a divergence of $\gamma^{-2}$. Also, $\Lambda_{l_{3}}\left(z_{3}\right) \equiv \Lambda_{k_{2}-k_{1}}\left(z_{3}\right)$ is of order unity as $\gamma \rightarrow 0$ since there are no roots with $\operatorname{Im}(z) \geq 0$ for wavenumbers other than $k_{2}$ and $k_{1}$; hence the $\Gamma_{3}$ terms in (69) - (70) are sub-dominant. The second term in (73) exhibits only the singularities of $\Gamma_{3}$ and may be dropped, while the second term in (72) has an additional factor with a pinching singularity similar to $\Gamma_{3}$, except that the roles of $z_{1}$ and $z_{3}$ are reversed, and gives an overall divergence of at most $\gamma^{-4}\left(\right.$ when $\left.v_{2}=v_{1}\right)$. The remaining terms in $(72)-(73)$ are

$$
\begin{aligned}
& <\partial_{v} \tilde{\psi}_{1}, \frac{\kappa \cdot I_{3}^{*}}{l_{3}\left(v-z_{3}^{*}\right)}>=<\partial_{v} \tilde{\psi}_{1}, \frac{\kappa^{2} \cdot \partial_{v} \psi_{1}}{k_{2} l_{3}\left(v-z_{3}^{*}\right)}>-<\partial_{v} \tilde{\psi}_{1}, \frac{\kappa^{2} \cdot \partial_{v} \psi_{2}^{*}}{k_{1} l_{3}\left(v-z_{3}^{*}\right)}> \\
& <\partial_{v} \tilde{\psi}_{2}, \frac{\kappa \cdot I_{3}}{l_{3}\left(v-z_{3}\right)}>=<\partial_{v} \tilde{\psi}_{2}, \frac{\kappa^{2} \cdot \partial_{v} \psi_{1}^{*}}{k_{2} l_{3}\left(v-z_{3}\right)}>-<\partial_{v} \tilde{\psi}_{2}, \frac{\kappa^{2} \cdot \partial_{v} \psi_{2}}{k_{1} l_{3}\left(v-z_{3}\right)}>;
\end{aligned}
$$

in all cases there are five poles in the integrand and hence a maximum possible $\gamma^{-4}$ divergence. A closer examination shows that, if $v_{2}=v_{1}$, this singularity is realized by the first term in (76) and both terms in (75); in any event we do not encounter a singularity in $r_{2}(0)$ or $p_{1}(0)$ 
that dominates those found at third order in the single mode equations, i.e. a singularity stronger than $\gamma^{-4}$.

When $k_{2}=2 k_{1}, \Lambda_{l_{3}}\left(z_{3}\right) \equiv \Lambda_{k_{1}}\left(z_{3}\right)$ with

$$
z_{3}=\left(2 v_{2}-v_{1}\right)+i\left(\frac{2 \gamma}{k_{1}}\right)
$$

Thus when $v_{2}=v_{1}$ we have $z_{3}=z_{1}+i \gamma / k_{1}$, and $\Lambda_{l_{3}}\left(z_{3}\right)$ is $\mathcal{O}(\gamma)$ as $\gamma \rightarrow 0^{+}$; if $v_{2} \neq v_{1}, \Lambda_{l_{3}}\left(z_{3}\right)$ is still of order one. Thus with equal phase velocities, the singularity of $\Gamma_{3}$ is increased to $\gamma^{-3}$ (including the presence of the third term in (71) which has a $\gamma^{-2}$ divergence in $s(0)$ ); nevertheless the $\Gamma_{3}$ terms in (69) - (70) are still sub-dominant. The second term in (73) can be neglected for similar reasons. However, the second term in (72) now has an apparent divergence of $\gamma^{-5}$ when $v_{2}=v_{1}$; such a singularity would not be absorbed by the scalings used to remove the $\gamma^{-4}$ singularities characteristic of the single mode problem. We will evaluate this term more precisely shortly. The first terms in (72) - (73) contain extra contributions from the third term in (71):

$$
\begin{gathered}
<\partial_{v} \tilde{\psi}_{1}, \frac{\kappa \cdot I_{3}^{*}}{l_{3}\left(v-z_{3}^{*}\right)}>=<\partial_{v} \tilde{\psi}_{1}, \frac{\kappa^{2} \cdot \partial_{v} \psi_{1}}{k_{2} l_{3}\left(v-z_{3}^{*}\right)}>-<\partial_{v} \tilde{\psi}_{1}, \frac{\kappa^{2} \cdot \partial_{v} \psi_{2}^{*}}{k_{1} l_{3}\left(v-z_{3}^{*}\right)}> \\
-i s(0)^{*}<\partial_{v} \tilde{\psi}_{1}, \frac{\kappa \cdot \psi_{1}^{*}}{l_{3}\left(v-z_{3}^{*}\right)}> \\
<\partial_{v} \tilde{\psi}_{2}, \frac{\kappa \cdot I_{3}}{l_{3}\left(v-z_{3}\right)}>=<\partial_{v} \tilde{\psi}_{2}, \frac{\kappa^{2} \cdot \partial_{v} \psi_{1}^{*}}{k_{2} l_{3}\left(v-z_{3}\right)}>-<\partial_{v} \tilde{\psi}_{2}, \frac{\kappa^{2} \cdot \partial_{v} \psi_{2}}{k_{1} l_{3}\left(v-z_{3}\right)}> \\
+i s(0)<\partial_{v} \tilde{\psi}_{2}, \frac{\kappa \cdot \psi_{1}}{l_{3}\left(v-z_{3}\right)}>.
\end{gathered}
$$

Our previous discussion of the first two terms in (78) and (79) is unchanged, and the new term in (79) shows only the $\gamma^{-2}$ singularity in $s(0)$ (as determined from equation (87) below). This singularity is also present in the third term of (78), but now $s(0)^{*}$ multiplies an integral that has an apparent singularity of $\gamma^{-3}$ giving a second term with overall $\gamma^{-5}$ divergence.

The foregoing discussion shows that there are new singularities in the coupling coefficients and that these singularities are most severe when $k_{2}=2 k_{1}$ and $v_{1}=v_{2}$. In this resonant 
case, the $p_{1}(0)$ singularity never exceeds $\gamma^{-4}$, but we have identified two contributions to $<\partial_{v} \tilde{\psi}_{1}, h_{3}^{*}>$ in the cubic coefficient $r_{2}(0)$ whose singularities appear to dominate the $\gamma^{-4}$ divergence characteristic of the cubic terms of the single mode problem. We proceed to a detailed evaluation of these exceptional terms which shows that the $\gamma^{-5}$ singularities are typically present.

For the second term in (72) the calculation of the integrals using partial fraction expansions (cf. (I)) yields the following asymptotic forms when $v_{2}=v_{1}$ :

$$
\begin{aligned}
& \Gamma_{3}=\frac{1}{\gamma^{3}}\left[\left(\frac{2 \pi k_{1}^{2}}{9 \Lambda_{k_{1}}^{\prime}\left(v_{1}\right)}\right) \sum_{s}^{\prime} \kappa^{(s)}\left(1+\kappa^{(s)}\right) \eta_{k_{1}}^{(s)}\left(v_{1}\right)+\mathcal{O}(\gamma)\right] \\
&<\partial_{v} \tilde{\psi}_{1}, \frac{\kappa^{2} \cdot \eta_{l_{3}}}{\left(v-z_{3}^{*}\right)}>=\frac{1}{\gamma^{2}}\left[\left(\frac{2 \pi i k_{1}^{2}}{9 \Lambda_{k_{1}}^{\prime}\left(v_{1}\right)}\right) \sum_{s}^{\prime} \kappa^{(s)}\left(1+\kappa^{(s)}\right) \eta_{k_{1}}^{(s)}\left(v_{1}\right)+\mathcal{O}(\gamma)\right]
\end{aligned}
$$

where the primed species summation excludes the electrons. In writing (81) we have used the dispersion relation (16). Given the assumption (67) on the single mode problem, we expect $\sum_{s}{ }^{\prime} \kappa^{(s)}\left(1+\kappa^{(s)}\right) \eta_{k_{1}}^{(s)}\left(v_{1}\right) \neq 0$ to typically hold; hence the $\gamma^{-5}$ singularity is generally present. Finally, the third term in (78) contains a $\gamma^{-2}$ singularity from $s(0)^{*}$ (equation (87)) while a partial fraction expansion of the integral yields

$$
<\partial_{v} \tilde{\psi}_{1}, \frac{\kappa \cdot \psi_{1}^{*}}{\left(v-z_{3}^{*}\right)}>=\frac{1}{\gamma^{3}}\left[-\left(\frac{5 \pi k_{1}^{3}}{18 \Lambda_{k_{1}}^{\prime}\left(v_{1}\right)}\right) \sum_{s}^{\prime} \kappa^{(s)}\left(1+\kappa^{(s)}\right) \eta_{k_{1}}^{(s)}\left(v_{1}\right)+\mathcal{O}(\gamma)\right]
$$

Thus the third term in (178) also realizes a $\gamma^{-5}$ singularity. These singularities require a shift in the scaling exponents that characterize the single mode instability; this point is discussed below in Section 4 .

\subsection{2 $\quad F_{0}(v)=F_{0}(-v)$, real eigenvalues}

For reflection-symmetric instabilities with real eigenvalues (Section 2.2.1), we have $v_{1}=$ $v_{2}=0$ and $k_{2}+k_{1}>0$ for two positive and unequal wavenumbers. The previous analysis is applicable here and we obtain the same conclusions with one modification. Since the 
Table 1: Generic singularities of the couplings and scaling exponents

\begin{tabular}{|c|c|c|c|c|c|c|c|c|}
\hline Instability & Resonance & $q$ & $s$ & $r_{1}^{\dagger}$ & $r_{2}$ & $p_{1}$ & $p_{2}^{\dagger}$ & $\left(\beta_{1}, \beta_{2}\right)$ \\
\hline$F_{0}(v) \neq F_{0}(-v)$ & $k_{2} \neq 2 k_{1}, v_{2} \neq v_{1}$ & - & - & $\gamma^{-4}$ & $\gamma^{-1}$ & $\gamma^{-1}$ & $\gamma^{-4}$ & $\left(\frac{5}{2}, \frac{5}{2}\right)$ \\
\hline \multirow{5}{*}{$\begin{array}{l}k_{2}>k_{1}>0 \\
\lambda_{1}, \lambda_{2} \text { complex }\end{array}$} & $v_{2}=v_{1}$ & - & - & $\gamma^{-4}$ & $\gamma^{-4}$ & $\gamma^{-4}$ & $\gamma^{-4}$ & $\left(\frac{5}{2}, \frac{5}{2}\right)$ \\
\hline & $k_{2}=2 k_{1}, v_{2} \neq v_{1}$ & $\mathcal{O}(1)$ & $\gamma^{-2}$ & $\gamma^{-4}$ & $\gamma^{-2}$ & $\gamma^{-2}$ & $\gamma^{-4}$ & $\left(\frac{5}{2}, \frac{5}{2}\right)$ \\
\hline & $v_{2}=v_{1}$ & $\mathcal{O}(1)$ & $\gamma^{-2}$ & $\gamma^{-4}$ & $\gamma^{-5}$ & $\gamma^{-4}$ & $\gamma^{-4}$ & $\left(\frac{5}{2}, 3\right)$ \\
\hline & $k_{2}=3 k_{1}, v_{2} \neq v_{1}$ & $\mathcal{O}(1)$ & $\gamma^{-4}$ & $\gamma^{-4}$ & $\gamma^{-1}$ & $\gamma^{-1}$ & $\gamma^{-4}$ & $\left(\frac{5}{2}, \frac{5}{2}\right)$ \\
\hline & $v_{2}=v_{1}$ & $\mathcal{O}(1)$ & $\gamma^{-4}$ & $\gamma^{-4}$ & $\gamma^{-4}$ & $\gamma^{-4}$ & $\gamma^{-4}$ & $\left(\frac{5}{2}, \frac{5}{2}\right)$ \\
\hline \multirow{3}{*}{$\begin{array}{l}F_{0}(v)=F_{0}(-v) \\
k_{2}>k_{1}>0 \\
\lambda_{1}, \lambda_{2} \text { real, } \\
\text { multiplicity-two }\end{array}$} & $k_{2} \neq 2 k_{1}$ & - & - & $\gamma^{-4}$ & $\gamma^{-4}$ & $\gamma^{-4}$ & $\gamma^{-4}$ & $\left(\frac{5}{2}, \frac{5}{2}\right)$ \\
\hline & $k_{2}=2 k_{1}$ & $\mathcal{O}(1)$ & $\gamma^{-2}$ & $\gamma^{-4}$ & $\gamma^{-5}$ & $\gamma^{-4}$ & $\gamma^{-4}$ & $\left(\frac{5}{2}, 3\right)$ \\
\hline & $k_{2}=3 k_{1}$ & $\mathcal{O}(1)$ & $\gamma^{-4}$ & $\gamma^{-4}$ & $\gamma^{-4}$ & $\gamma^{-4}$ & $\gamma^{-4}$ & $\left(\frac{5}{2}, \frac{5}{2}\right)$ \\
\hline
\end{tabular}

$\dagger=$ single mode

condition $v_{1}=v_{2}$ is automatically satisfied, the "spatial resonance", $k_{2}=2 k_{1}$, is sufficient to obtain the extra singularities noted above. These results are summarized in Table 1 .

\subsection{3 $\quad F_{0}(v)=F_{0}(-v)$, complex eigenvalues}

For reflection-symmetric instabilities with complex eigenvalues (Section 2.2.2), we have $v_{1}=$ $-v_{2}$ and $k_{2}+k_{1}=0$. These conditions rule out the presence of $k_{2}=2 k_{1}$ or $k_{2}=3 k_{1}$ resonances, and imply that $p_{1}(0)=r_{2}(0)$, where

$$
p_{1}(0)=-i\left[\frac{<\partial_{v} \tilde{\psi}_{2}, \kappa \cdot\left(h_{3}-h_{5}\right)>}{k_{1}}+\frac{<\partial_{v} \tilde{\psi}_{2}, \kappa \cdot h_{1}>}{k_{2}}+\frac{\Gamma_{3}<\partial_{v} \tilde{\psi}_{2}, \kappa \cdot \psi_{1}>}{k_{2}-k_{1}}\right]
$$

with $h_{5}$ defined in (60).

From $z_{1}=-z_{2}=z_{0}$ we now have $z_{1}$ in the upper-half plane $\left(k_{1}>0\right)$ and $z_{2}$ in the lower-half plane $\left(k_{2}<0\right)$ along with $z_{3}=-i \gamma / k_{1}$. The eigenfunctions $\psi_{1}(v)$ and $\tilde{\psi}_{1}(v)^{*}$ 
have poles in the upper-half plane that approach $v_{1}$ as $\gamma \rightarrow 0^{+}$, while the poles of $\psi_{2}(v)$ and $\tilde{\psi}_{2}(v)^{*}$ are in the lower half-plane and approach $v_{2}$ as $\gamma \rightarrow 0^{+}$. The integrals $<\partial_{v} \tilde{\psi}_{1}, \kappa \cdot \psi_{2}>$ and $<\partial_{v} \tilde{\psi}_{2}, \kappa \cdot \psi_{1}>$ are still nonsingular although they now involve poles above and below the contour. The relation $v_{1}=-v_{2}$ ensures that no pinching singularity develops. Similarly, $I_{5}(v)$ and $h_{5}(v)$ have poles on each side of the contour but no pinch can develop in the integrals $<\partial_{v} \tilde{\psi}_{1}, \kappa \cdot h_{5}>$ and $<\partial_{v} \tilde{\psi}_{2}, \kappa \cdot h_{5}>$. In

$$
<\partial_{v} \tilde{\psi}_{1}, \kappa \cdot h_{2}>=\frac{i}{2 \gamma k_{2}}\left[<\partial_{v} \tilde{\psi}_{1}, \kappa^{2} \cdot \partial_{v} \psi_{2}^{*}>-<\partial_{v} \tilde{\psi}_{1}, \kappa^{2} \cdot \partial_{v} \psi_{2}>\right]
$$

the first integral $<\partial_{v} \tilde{\psi}_{1}, \kappa^{2} \cdot \partial_{v} \psi_{2}^{*}>$ only has poles in the upper-half plane while the second integral has poles above and below the contour but no pinch; thus this term may also be dropped as well as the corresponding term $<\partial_{v} \tilde{\psi}_{2}, \kappa \cdot h_{1}>$ in $p_{1}(0)$.

Discarding these manifestly nonsingular terms, we must still reconsider the remaining terms in (69) - (73). The prior analysis given in Section 3.1.1 requires relatively minor modifications to allow for the relation $v_{1}=-v_{2}$ and the shifted location of the poles $z_{2}$ and $z_{3}$. In (71),$\Lambda_{l_{3}}\left(z_{3}\right) \equiv \Lambda_{-2 k_{1}}\left(z_{3}\right)$ is of order one as $\gamma \rightarrow 0^{+}$and the integrals are free of pinching singularities since $z_{3}$ is pure imaginary and can never converge to either $z_{1}$ or $z_{2}$. Thus the second terms in $([69)$ - (70) are nonsingular as are the second terms in (72) (73). The final integrals in the first terms of $(72)-(73)$, written out in (75) - (76), are also manifestly free of pinching singularities. In summary, the coupling coefficients $p_{1}(0), r_{2}(0)$ for instabilities with complex coefficients and reflection-symmetry are identical, and exhibit singularities due to the explicit $\gamma^{-1}$ factor in $\left\langle\partial_{v} \tilde{\psi}_{1}, \kappa \cdot h_{2}>\right.$ only.

\subsection{The couplings $Q_{1}(0), P_{2}(0), Q_{3}(0)$ and $Q_{1}(0)+P_{3}(0)$}

For reflection-symmetric instabilities with complex eigenvalues (Section 2.2.2), there are four additional $\mathrm{O}(2)$ symmetric couplings at third order. Although these terms can be removed 
Table 2: Generic singularities of the couplings and scaling exponents

\begin{tabular}{lccccccccc} 
Instability & $Q_{1}$ & $P_{2}$ & $Q_{3}$ & $\left(Q_{1}+P_{3}\right)$ & $r_{1}^{\dagger}$ & $r_{2}$ & $p_{1}$ & $p_{2}^{\dagger}$ & $\left(\beta_{1}, \beta_{2}\right)$ \\
\hline$F_{0}(v)=F_{0}(-v)$ & $\gamma^{-1}$ & $\mathcal{O}(1)$ & $\gamma^{-3}$ & $\gamma^{-4}$ & $\gamma^{-4}$ & $\gamma^{-1}$ & $\gamma^{-1}$ & $\gamma^{-4}$ & $\left(\frac{5}{2}, \frac{5}{2}\right)$ \\
$k_{2}+k_{1}=0$ & & & & & & & & & \\
$\lambda_{1}$ complex, & & & & & & & & & \\
multiplicity-two & & & & & & & & & \\
\hline
\end{tabular}

$\dagger=$ single mode

by a coordinate transformation to obtain the normal form in (38), it is important to consider their asymptotic behavior.

The singularities for the couplings in this instablity are summarized in Table 2. $P_{2}(0)$ is nonsingular and the singularities of $Q_{1}(0)$ and $Q_{3}(0)$ are sub-dominant. The strongest singularity occurs in $Q_{1}(0)+P_{3}(0)$ due the integral

$$
<\partial_{v} \tilde{\psi}_{1}, \kappa \cdot h_{1}>=\frac{i}{2 \gamma k_{1}}\left[<\partial_{v} \tilde{\psi}_{1}, \kappa^{2} \cdot \partial_{v} \psi_{1}^{*}>-<\partial_{v} \tilde{\psi}_{1}, \kappa^{2} \cdot \partial_{v} \psi_{1}>\right]
$$

whose first term $<\partial_{v} \tilde{\psi}_{1}, \kappa^{2} \cdot \partial_{v} \psi_{1}^{*}>$ has a $\gamma^{-3}$ singularity giving an overall singularity of $\gamma^{-4}$ for $Q_{1}(0)+P_{3}(0)$.

\subsection{The spatial resonances: $q(0)$ and $s(0)$}

When $k_{2}=2 k_{1}$ and $k_{2}=3 k_{1}$ there are additional couplings at second and third order, respectively. We first consider $q(0)$ and $s(0)$ for the $k_{2}=2 k_{1}$ resonance, noting that the two integrals, $<\partial_{v} \tilde{\psi}_{1}, \kappa \cdot \psi_{2}>$ and $<\partial_{v} \tilde{\psi}_{2}, \kappa \cdot \psi_{1}>$, have poles at $z_{1}$ and $z_{2}$ and are free of pinching singularities in all cases. Thus $q(0)$ is nonsingular as is the second term in $s(0)$. The first term in $s(0)$ was evaluated in (I),

$$
<\partial_{v} \tilde{\psi}_{1}, \kappa \cdot \psi_{1}^{*}>=\left(\frac{i k_{1}}{2 \gamma_{1}}\right)^{2}\left[\frac{2 i}{\Lambda_{k_{1}}^{\prime}\left(z_{1}\right)} \sum_{s}^{\prime} \kappa^{(s)}\left(1+\kappa^{(s)}\right) \operatorname{Im} \int_{-\infty}^{\infty} \frac{d v \eta_{k_{1}}^{(s)}}{v-z_{1}}+\mathcal{O}\left(\gamma_{1}\right)\right]
$$


where the primed species sum omits the electrons; this determines the singularity of $s(0)$,

$$
s(0)=\frac{1}{\gamma^{2}}\left[-\frac{\pi k_{1}^{2}}{2 \Lambda_{k_{1}}^{\prime}\left(z_{1}\right)} \sum_{s}^{\prime} \kappa^{(s)}\left(1+\kappa^{(s)}\right) \eta_{k_{1}}^{(s)}\left(v_{1}\right)+\mathcal{O}(\gamma)\right] .
$$

For the $k_{2}=3 k_{1}$ resonance $q(0)$ is again readily seen to be nonsingular and we omit the details. For $s(0)$ in (44) the integral $<\partial_{v} \tilde{\psi}_{1}, \kappa \cdot h_{4}^{*}>$ contains a contribution,

$$
<\partial_{v} \tilde{\psi}_{1}, \frac{\kappa \cdot I_{4}^{*}}{v-z_{1}^{*}}>\sim \mathcal{O}\left(\gamma^{-4}\right)
$$

if $v_{2} \neq v_{1}$. When $v_{2}=v_{1}$ there are additional singularities, but none stronger than $\gamma^{-4}$. These conclusions are summarized in Table 1.

\subsection{Special limits: coupling singularities with fixed ions}

In the various explicit asymptotic formulas, such as (64), (80) - (82), and (87), the leading term vanishes if the ion masses are treated as infinite since $\kappa^{(s)} \rightarrow 0$ in this limit. In (I), this suppression of the leading singularity was shown to be a general feature of the integrals that appearing in the amplitude equations. For the single mode instability, the cubic coefficient $p_{1}(0)$ has a $\gamma^{-3}$ singularity when the ions are fixed, and the modified single-mode scaling $A(t)=\gamma^{2} a(\gamma t)$ suffices to render the amplitude expansion finite. [1, 3]

It is straightforward to adapt the results of the previous sections to the case of infinitely massive ions: with only a few exceptions among terms that are already sub-dominant the generic divergence is reduced by one factor of $\gamma^{-1}$. For our purposes it is suffices to summarize in Table 3 the resulting changes to Table 1 when the electrons are the only mobile species.

\section{Nonlinear scalings}

In this section we make use of the leading order behavior of the coupling coefficients identified in the preceding section to determine the scaling of the saturation amplitude of the two 
Table 3: Summary of the singularities of the couplings for fixed ions

\begin{tabular}{|c|c|c|c|c|c|c|c|c|}
\hline Instability & Resonance & $q$ & $s$ & $r_{1}^{\dagger}$ & $r_{2}$ & $p_{1}$ & $p_{2}^{\dagger}$ & $\left(\beta_{1}, \beta_{2}\right)$ \\
\hline$F_{0}(v) \neq F_{0}(-v)$ & $k_{2} \neq 2 k_{1}, v_{2} \neq v_{1}$ & - & - & $\gamma^{-3}$ & $\gamma^{-1}$ & $\gamma^{-1}$ & $\gamma^{-3}$ & $(2,2)$ \\
\hline \multirow{5}{*}{$\begin{array}{l}k_{2}>k_{1}>0 \\
\lambda_{1}, \lambda_{2} \text { complex }\end{array}$} & $v_{2}=v_{1}$ & - & - & $\gamma^{-3}$ & $\gamma^{-3}$ & $\gamma^{-3}$ & $\gamma^{-3}$ & $(2,2)$ \\
\hline & $k_{2}=2 k_{1}, v_{2} \neq v_{1}$ & $\mathcal{O}(1)$ & $\gamma^{-1}$ & $\gamma^{-3}$ & $\gamma^{-1}$ & $\gamma^{-1}$ & $\gamma^{-3}$ & $(2,2)$ \\
\hline & $v_{2}=v_{1}$ & $\mathcal{O}(1)$ & $\gamma^{-1}$ & $\gamma^{-3}$ & $\gamma^{-4}$ & $\gamma^{-3}$ & $\gamma^{-3}$ & $\left(2, \frac{5}{2}\right)$ \\
\hline & $k_{2}=3 k_{1}, v_{2} \neq v_{1}$ & $\mathcal{O}(1)$ & $\gamma^{-3}$ & $\gamma^{-3}$ & $\gamma^{-1}$ & $\gamma^{-1}$ & $\gamma^{-3}$ & $(2,2)$ \\
\hline & $v_{2}=v_{1}$ & $\mathcal{O}(1)$ & $\gamma^{-3}$ & $\gamma^{-3}$ & $\gamma^{-3}$ & $\gamma^{-3}$ & $\gamma^{-3}$ & $(2,2)$ \\
\hline \multirow{3}{*}{$\begin{array}{l}F_{0}(v)=F_{0}(-v) \\
k_{2}>k_{1}>0 \\
\lambda_{1}, \lambda_{2} \text { real, } \\
\text { multiplicity-two }\end{array}$} & $k_{2} \neq 2 k_{1}$ & - & - & $\gamma^{-3}$ & $\gamma^{-3}$ & $\gamma^{-3}$ & $\gamma^{-3}$ & $(2,2)$ \\
\hline & $k_{2}=2 k_{1}$ & $\mathcal{O}(1)$ & $\gamma^{-1}$ & $\gamma^{-3}$ & $\gamma^{-4}$ & $\gamma^{-3}$ & $\gamma^{-3}$ & $\left(2, \frac{5}{2}\right)$ \\
\hline & $k_{2}=3 k_{1}$ & $\mathcal{O}(1)$ & $\gamma^{-3}$ & $\gamma^{-3}$ & $\gamma^{-3}$ & $\gamma^{-3}$ & $\gamma^{-3}$ & $(2,2)$ \\
\hline
\end{tabular}

$\dagger=$ single mode

competing modes with the growth rate $\gamma$. We first consider this question for the coefficient singularities listed in Table 1, i.e. for instabilities with $k_{2}>k_{1}>0$. Following (I), we introduce scaled amplitudes $A(t) \equiv \gamma^{\beta_{1}} a(\gamma t) \exp \left(-i \theta_{1}(t)\right)$ and $B(t) \equiv \gamma^{\beta_{2}} a(\gamma t) \exp \left(-i \theta_{2}(t)\right)$ for $\gamma>0$ and rewrite (24) - (25) using the expansions in (41) and (49):

$$
\begin{gathered}
\frac{d a}{d \tau}=a+\gamma^{\beta_{2}-1} \operatorname{Re}\left[s(0) e^{i\left(2 \theta_{1}-\theta_{2}\right)}\right] a b \delta_{k_{2}, 2 k_{1}}+\gamma^{2 \beta_{1}-1} \operatorname{Re}\left[r_{1}(0)\right] a^{3} \\
+\gamma^{2 \beta_{2}-1} \operatorname{Re}\left[r_{2}(0)\right] a b^{2}+\gamma^{\beta_{2}+\beta_{1}-1} \operatorname{Re}\left[s(0) e^{i\left(3 \theta_{1}-\theta_{2}\right)}\right] a^{2} b \delta_{k_{2}, 3 k_{1}}+\cdots \\
\frac{d b}{d \tau}=b+\gamma^{2 \beta_{1}-\beta_{2}-1} \operatorname{Re}\left[q(0) e^{-i\left(2 \theta_{1}-\theta_{2}\right)}\right] a^{2} \delta_{k_{2}, 2 k_{1}}+\gamma^{2 \beta_{1}-1} \operatorname{Re}\left[p_{1}(0)\right] a^{2} b \\
+\gamma^{3 \beta_{1}-\beta_{2}-1} \operatorname{Re}\left[q(0) e^{-i\left(3 \theta_{1}-\theta_{2}\right)}\right] a^{3} \delta_{k_{2}, 3 k_{1}}+\gamma^{2 \beta_{2}-1} \operatorname{Re}\left[p_{2}(0)\right] b^{3}+\cdots \\
\frac{d \theta_{1}}{d t}=\omega_{1}-\gamma^{\beta_{2}} \operatorname{Im}\left[s(0) e^{i\left(2 \theta_{1}-\theta_{2}\right)}\right] b \delta_{k_{2}, 2 k_{1}}-\gamma^{2 \beta_{1}} \operatorname{Im}\left[r_{1}(0)\right] a^{2} \\
-\gamma^{2 \beta_{2}} \operatorname{Im}\left[r_{2}(0)\right] b^{2}-\gamma^{\beta_{2}+\beta_{1}} \operatorname{Im}\left[s(0) e^{i\left(3 \theta_{1}-\theta_{2}\right)}\right] a b \delta_{k_{2}, 3 k_{1}}+\cdots
\end{gathered}
$$




$$
\begin{aligned}
\frac{d \theta_{2}}{d t}=\omega_{2}-\gamma^{2 \beta_{1}-\beta_{2}} & \operatorname{Im}\left[q(0) e^{-i\left(2 \theta_{1}-\theta_{2}\right)}\right] \frac{a^{2}}{b} \delta_{k_{2}, 2 k_{1}}-\gamma^{2 \beta_{1}} \operatorname{Im}\left[p_{1}(0)\right] a^{2} \\
& -\gamma^{2 \beta_{2}} \operatorname{Im}\left[p_{2}(0)\right] b^{2}-\gamma^{3 \beta_{1}-\beta_{2}} \operatorname{Im}\left[q(0) e^{-i\left(3 \theta_{1}-\theta_{2}\right)}\right] \frac{a^{3}}{b} \delta_{k_{2}, 3 k_{1}}+\cdots
\end{aligned}
$$

If possible, the choice of $\beta_{1}$ and $\beta_{2}$ should be made so that each term is finite as $\gamma \rightarrow 0^{+}$and there is a formal balance between linear and nonlinear terms in (89) - (90).

In Table 1 , we focus initially on the instabilities with $k_{2} \neq 2 k_{1}$. The $\gamma^{-4}$ singularities of the single mode coefficients $r_{1}(0)$ and $p_{2}(0)$ in (89) - (90) require $\beta_{1} \geq 5 / 2$ and $\beta_{2} \geq 5 / 2$. These exponents are large enough to ensure a finite limit for each term (91) - (92); in fact the phase equations reduce to $\theta_{j}=\omega_{j}+\mathcal{O}(\gamma), j=1,2$. In (89) - (90), the minimal choice $\beta_{j}=5 / 2$ suffices to control the singularities in the mode couplings $r_{2}(0)$ and $p_{1}(0)$ as well and the amplitude equations reduce to

$$
\begin{aligned}
& \frac{d a}{d \tau}=a+\operatorname{Re}\left[c\left(k_{1}, v_{1}+i 0\right)\right] a^{3}+\gamma^{4} \operatorname{Re}\left[r_{2}(0)\right] a b^{2}+\gamma^{4} \operatorname{Re}\left[s(0) e^{i\left(3 \theta_{1}-\theta_{2}\right)}\right] a^{2} b \delta_{k_{2}, 3 k_{1}}+\cdots(\cdot 93) \\
& \frac{d b}{d \tau}=b+\gamma^{4} \operatorname{Re}\left[p_{1}(0)\right] a^{2} b+\operatorname{Re}\left[c\left(k_{2}, v_{2}+i 0\right)\right] b^{3}+\gamma^{4} \operatorname{Re}\left[q(0) e^{-i\left(3 \theta_{1}-\theta_{2}\right)}\right] a^{3} \delta_{k_{2}, 3 k_{1}}+\cdots(.94)
\end{aligned}
$$

where $c\left(k_{j}, z_{j}\right)$ is defined in (65). In these variables the linear growth rates are unity and the single mode terms, $a^{3}$ and $b^{3}$, are of $\mathcal{O}(1)$ in $\gamma$. If $v_{2}=v_{1}$, then the coupling coefficients $\gamma^{4} r_{2}(0)$ and $\gamma^{4} p_{1}(0)$ are also of $\mathcal{O}(1)$, but otherwise these terms are sub-dominant. When it is present, the resonant term, $\gamma^{4} s(0) e^{i\left(3 \theta_{1}-\theta_{2}\right)}$, is formally of $\mathcal{O}(1)$; however the phases in the exponential evolve on the fast time scale $t=\tau / \gamma$ and therefore the exponential oscillates very rapidly as $\gamma \rightarrow 0^{+}$unless the linear frequencies are resonant: $3 \omega_{1}=\omega_{2}$. Such a rapid oscillation allows the term to be time-averaged and neglected, but when $3 \omega_{1}=\omega_{2}$ the phase is stationary and this $\mathcal{O}(1)$ term cannot be discarded by time-averaging. Note that, given the spatial resonance $k_{2}=3 k_{1}$, the frequency resonance is equivalent to assuming that the linear phase velocities are equal $v_{2}=v_{1}$. The second resonant term, $\gamma^{4} q(0) e^{-i\left(3 \theta_{1}-\theta_{2}\right)}$, is formally of $\mathcal{O}\left(\gamma^{4}\right)$ but nevertheless has a qualitatively important effect on the dynamics near $b=0$ as 
discussed below. Thus the asymptotic equations (93) - (94) are correct when $v_{2}=v_{1}$, but if $v_{2} \neq v_{1}$ then their form simplifies to

$$
\begin{aligned}
& \frac{d a}{d \tau}=a+\operatorname{Re}\left[c\left(k_{1}, v_{1}+i 0\right)\right] a^{3}+\cdots \\
& \frac{d b}{d \tau}=b+\operatorname{Re}\left[c\left(k_{2}, v_{2}+i 0\right)\right] b^{3}+\gamma^{4} \operatorname{Re}\left[q(0) e^{-i\left(3 \theta_{1}-\theta_{2}\right)}\right] a^{3} \delta_{k_{2}, 3 k_{1}}+\cdots
\end{aligned}
$$

this system describes the evolution of the waves as if they were non-interacting, indeed their mutual dynamics is essentially the "superposition" of the two single mode amplitude equations save for the very weak resonant term in (96). For $k_{2} \neq 2 k_{1}$, our principal conclusion is that no change from the scaling exponents predicted by the single mode singularities is indicated.

The resonance $k_{2}=2 k_{1}$ is special: the $s(0)$ resonant term now occurs at second order with a $\gamma^{-2}$ singularity and, if $v_{2}=v_{1}$, the singularity in $r_{2}(0)$ jumps to $\gamma^{-5}$. In addition, $v_{2}=v_{1}$ implies a frequency resonance $2 \omega_{1}=\omega_{2}$ that suppresses the oscillation of the quadratic term. If we keep $\beta_{2}=5 / 2$ in (89), then this quadratic term retains a $\gamma^{-1 / 2}$ singularity that cannot be erased by time averaging. Similarly the cubic term $\gamma^{4} \operatorname{Re}\left[r_{2}(0)\right] a b^{2}$ would retain a $\gamma^{-1}$ singularity. In this situation, the scaling exponent for the short wavelength mode must be increased to $\beta_{2}=3$ to obtain a sensible asymptotic limit. Now the amplitude equations reduce to

$$
\begin{aligned}
& \frac{d a}{d \tau}=a+\gamma^{2} \operatorname{Re}\left[s(0) e^{i\left(2 \theta_{1}-\theta_{2}\right)}\right] a b+\operatorname{Re}\left[c\left(k_{1}, v_{1}+i 0\right)\right] a^{3}+\gamma^{5} \operatorname{Re}\left[r_{2}(0)\right] a b^{2}+\cdots \\
& \frac{d b}{d \tau}=b+\gamma \operatorname{Re}\left[q(0) e^{-i\left(2 \theta_{1}-\theta_{2}\right)}\right] a^{2}+\gamma^{4} \operatorname{Re}\left[p_{1}(0)\right] a^{2} b+\cdots
\end{aligned}
$$

in these variables the growth rates are again unity and all terms shown are $\mathcal{O}(1)$ in $\gamma$ except for $\gamma \operatorname{Re}\left[q(0) \exp -i\left(2 \theta_{1}-\theta_{2}\right)\right] a^{2}$ which is $\mathcal{O}(\gamma)$ but is nevertheless important for the asymptotic dynamics as explained below. When $k_{2}=2 k_{1}$ but $v_{2} \neq v_{1}$, the quadratic term in (97) can be removed by time-averaging and the singularity of $r_{2}(0)$ and $p_{1}(0)$ drops to $\gamma^{-2}$; 
in this case the single mode scalings suffice to control the singularities in (89) - (90) and the amplitude equations simplify to

$$
\begin{aligned}
& \frac{d a}{d \tau}=a+\operatorname{Re}\left[c\left(k_{1}, v_{1}+i 0\right)\right] a^{3}+\cdots \\
& \frac{d b}{d \tau}=b+\gamma \operatorname{Re}\left[q(0) e^{-i\left(2 \theta_{1}-\theta_{2}\right)}\right] a^{2}+\operatorname{Re}\left[c\left(k_{2}, v_{2}+i 0\right)\right] b^{3}+\cdots
\end{aligned}
$$

In the resonant regime, $k_{2}=2 k_{1}$ and $v_{2}=v_{1}$, the long wavelength mode saturates at a $\mathcal{O}\left(\gamma^{5 / 2}\right)$ amplitude while the short wavelength mode saturates at a much smaller $\mathcal{O}\left(\gamma^{3}\right)$ amplitude, i.e. the presence of the long mode results in a dramatic suppression of the amplitude of the short mode. Because of the $\beta_{2}=3$ scaling required for the short wave mode the equation for $b(\tau)$ is dominated by the interaction with the long wave mode $a(\tau)$. In particular in the rescaled equation (98) the single mode terms appear at higher order and hence are omitted. On the other hand we have retained the $\mathcal{O}(\gamma)$ term $a^{2}$. Although formally small this term exerts a profound effect on the resulting dynamics. This is because it destroys the invariance of the subspace $b=0$. This term therefore plays the role of a "noise" term that continuously forces the short mode. This observation holds equally for the resonant $a^{3}$ term in (94).

The singularities summarized in Table 2 refer to an instability of a reflection-symmetric equilibrium involving complex eigenvalues. All the couplings in Table 2 are third order terms and none of the divergences are stronger than the $\gamma^{-4}$ singularities of the single mode couplings. Thus the single mode exponents $\beta_{j}=5 / 2$ will control all singularities in the amplitude equations to third order, and the rescaled equations have the form

$$
\begin{aligned}
& \frac{d a}{d \tau}=a+\operatorname{Re}\left[c\left(k_{1}, v_{1}+i 0\right)\right] a^{3}+\gamma^{4} \operatorname{Re}\left[e^{i\left(\theta_{b}+\theta_{a}\right)}\left(Q_{1}(0)+P_{3}(0)\right)\right] b a^{2}+\cdots \\
& \frac{d b}{d \tau}=b+\operatorname{Re}\left[c\left(k_{2}, v_{2}+i 0\right)\right] b^{3}+\gamma^{4} \operatorname{Re}\left[e^{i\left(\theta_{b}+\theta_{a}\right)}\left(Q_{1}(0)+P_{3}(0)\right)\right] a b^{2}+\cdots
\end{aligned}
$$

The joint conditions $k_{2}+k_{1}=0$ and $v_{2}=-v_{1}$ characteristic of this instability imply $\omega_{2}=\omega_{1}$; hence the exponentials in the mode coupling terms are rapidly oscillating and we expect these 
terms to have a negligible effect on the time-averaged evolution. In effect, the coupled system

(101) - (102) reduces to (95) - (96) and we recover the superposition picture of the two single mode amplitude equations but without the resonant $a^{3}$ term.

If the ions are taken to be infinitely massive, then Table 3 replaces Table 2 and the scaling associated with the single mode instabilities becomes $\beta_{j}=2$. The re-analysis of (89) - (90) leads similar conclusions although with quantitative differences. Only for the resonance $k_{2}=2 k_{1}$ and $v_{2}=v_{1}$ are the single mode scalings altered and again the short wavelength mode is suppressed; in this case we find $\beta_{2}$ increases to $\beta_{2}=5 / 2$. When $v_{2} \neq v_{1}$ we again find that the amplitude equations decouple as in (95) - (96).

\section{Discussion}

In this paper we have investigated the interaction between two weakly unstable electrostatic waves in an unmagnetized plasma. Such growing modes are defined unambiguously by eigenfunctions of the linear theory; Landau-damped collective modes are properly thought of as part of the continuum and hence are not decaying "modes" in the present sense. [6] For the growing modes discussed here, the theory provides a self-consistent description that includes the nonlinear effects of wave-particle resonance. Such a formulation, while more complex, captures phenomena that are absent from conventional formulations involving wave interactions. 11]

The wave-wave interaction is described by coupled amplitude equations consistent with the assumed translation invariance of the system and any symmetry of the unstable velocity distribution function. The coupling coefficients exhibit new singularities in the weakly unstable limit, but except in the case of the two-to-one spatial resonance do not alter the scaling for the saturation amplitude identified in single mode theory, at least through third order in the mode amplitudes. In the special but important case of two-to-one spatial resonance 
the overlap of velocity resonances and spatial resonance modifies the scaling, resulting in a dramatic suppression of the short wave mode.

In the absence of velocity overlap and the two-to-one spatial resonance the single mode scaling shows that the two waves evolve as a simple superposition of the individual instabilities. This picture is qualitatively consistent with the numerical results obtained by Demeio and Zweifel for beam-plasma instabilities with reflection symmetry. [10] In addition, it agrees more quantitatively with the analysis of Buchanan and Dorning who constructed superpositions of BGK modes as candidates for the asymptotic states produced in the numerical simulations. 12 In particular, these authors found that a consistent construction to leading order in the amplitude of the individual BGK modes required unequal phase velocities; an assumption analogous to our $v_{2} \neq v_{1}$ condition. Our results suggest a precise connection between the initial value problem for the unstable waves and the superposed BGK states of Buchanan and Dorning.

The significance of resonance overlap, such as $v_{2}=v_{1}$, in the single particle phase space is well-established from studies of particle motion in fields produced by large amplitude waves. In these studies the appearance of chaotic particle trajectories is investigated, but the selfconsistent modification of the wave evolution by the particles is routinely neglected. 13, [14, 15] The situation we consider does not allow this simplifying approximation since the resonant particles drive the linear instability and also dominate the nonlinear evolution of the waves. It is striking to discover that resonance ovelap has a profound effect on the dynamics of the waves, in addition to its better known consequences for the associated particle dynamics.

Our prediction that resonant interaction with a longer wavelength mode can modify the nonlinear scaling of a short wavelength mode may be amenable to experimental test. The single mode scaling for fixed ions was detected experimentally in measurements on an 
electron beam injected through a travelling wave tube. [16] The tube plays the role of the nonresonance electrons and supports a propagating wave which couples to the resonant particles in the beam. If the electron beam is sufficiently cold the unstable waves approximately satisfy the approximate dispersion relation $\omega(k)=v_{p} k$ with constant $v_{p}$ and hence always have equal phase velocities. [17] Under these conditions, one should measure the scaling of a single mode launched at $k_{2}$ and then repeat the measurement when a second wave is launched simultaneously at $k_{1}=k_{2} / 2$.

The possibility remains that singularities in the higher order coupling coefficients could modify these conclusions and force new scalings for other spatial resonances as well. However, in the study of singular amplitude equations in other problems it is commonly found that the dominant singularities appear in the low order nonlinear terms. [1], 18] In addition, the fact that the $k_{2}=3 k_{1}$ spatial resonance does not shift the scalings may signify that resonances other than $k_{2}=2 k_{1}$ will generally leave the single mode scalings undisturbed. These speculations can be tested by examining the singularities in the amplitude expansions to all orders. Such an investigation may be feasible using the techniques of (I).

\section{$6 \quad$ Acknowledgements}

This work supported by NSF grant PHY-9423583.

\section{References}

[1] J.D. Crawford and A. Jayaraman, Amplitude equations for electrostatic waves: multiple species, submitted to J. Math. Phys. Available from the Los Alamos archives at http://xxx.lanl.gov/abs/patt-sol/9706001. 
[2] J.D. Crawford and A. Jayaraman, Nonlinear saturation of an electrostatic wave: mobile ions modify trapping scaling, Phys. Rev. Lett. 77 3549-3552 (1996).

[3] J.D. Crawford, Amplitude equations for electrostatic waves: universal singular behavior in the limit of weak instability, Phys. Plasmas 2 97-128 (1995).

[4] N.G. van Kampen, On the theory of stationary waves in plasmas, Physica 21 (1955) 949-963; see also N.G. van Kampen and B.U. Felderhof, Theoretical Methods in Plasma Physics, (North-Holland, Amsterdam, 1967).

[5] K. Case, Plasma oscillations, Ann. Phys. 7 (1959) 349-364; also Plasma oscillations, Phys. Fl. 21 249-257 (1978).

[6] J.D. Crawford and P. Hislop, Application of the method of spectral deformation to the Vlasov-Poisson model, Ann. Phys. 189 265-317 (1989).

[7] M. Golubitsky, I. Stewart, and D.G. Schaeffer, Singularities and Groups in Bifurcation Theory: Vol. II, Appl. Math. Sci. 69, Springer-Verlag, New York (1988).

[8] J.D. Crawford and E. Knobloch, Symmetry and symmetry-breaking bifurcations in fluid dynamics, Annu. Rev. Fluid Mech. 23 (1991) 341-387.

[9] We assume that $n_{1}$ and $n_{2}$ have no common integer divisors other than unity; if this is not the case then the integers used in (30) - (32) are obtained from $n_{1}$ and $n_{2}$ by factoring out the common divisor.

[10] L. Demeio and P.F. Zweifel, Numerical simulations of perturbed Vlasov equilibria, Phys. Fl. 2B 1252-1255 (1990).

[11] M. Porkolab and R.P.H. Chang, Nonlinear wave effects in laboratory plasmas: a comparison between theory and experiment, Rev. Mod. Phys. 50 745-795 (1978). 
[12] M. Buchanan and J.J. Dorning, Superposition of nonlinear plasma waves, Phys. Rev. Lett. 70 3732-3735 (1993).

[13] B.V. Chirikov, A universal instability of many-dimensional oscillator systems, Phys. Rep. 52 (1979) 263-379.

[14] A.J. Lichtenberg and M.A. Lieberman, Regular and Stochastic Motion (Springer-Verlag, New York, 1983).

[15] D.F. Escande, Stochasticity in classical Hamiltonian systems: universal aspects, Phys. Rep. 121 (1985) 165-261.

[16] S.I. Tsunoda, F. Doveil, and J.H. Malmberg, Nonlinear interaction between a warm electron beam and a single wave, Phys. Rev. Lett. 59 2752-2755 (1987).

[17] J.D. Crawford, Appearance of inflection point modes in beam-plasma systems, Phys. Lett. A 209 (1995) 356-361.

[18] J.D. Crawford and K.T.R. Davies, Phase dynamical models of globally coupled oscillators: singularities and scaling with arbitrary coupling, (preprint, patt-sol/9701006 at LANL archives), submitted to Physica D. 